\title{
Una relación polar entre triángulos y circunferencias
}

\author{
Eduardo Velasco Barreras
}

\author{
Universidad de Sonora \\ e-mail: lalovelascobar@gmail.com
}

\begin{abstract}
Resumen
Utilizando rectas polares, presentamos una construcción por medio de la cual cada circunferencia en el plano de un triángulo induce puntos colineales sobre sus lados, y para algunas circunferencias especiales en el triángulo, estudiamos la recta determinada por dichos puntos. Presentamos además una caracterización de las familias de circunferencias que dan lugar a una misma recta. Finalmente, puntualizamos que nuestra construcción corresponde al campo de la geometría proyectiva, para dar paso a repetir la construcción utilizando secciones cónicas.
\end{abstract}

\section{Introducción}

La geometría es el estudio de las propiedades de las figuras y objetos en un espacio determinado. A lo largo de la historia de la humanidad, las diferentes civilizaciones han descubierto la importancia de la geometría, de acuerdo a las necesidades particulares de su tiempo. En sus inicios, la geometría consistía en resolver problemas concretos sobre el cálculo de longitudes, áreas y volúmenes. Hoy en día, las aplicaciones de la geometría están presentes en distintas áreas especializadas del conocimiento, del arte y de las ciencias. Esta evolución ha sido un proceso que consta de diferentes etapas en la historia.

El resultado que presentamos y desarrollamos en este trabajo puede catalogarse dentro de una rama de la geometría llamada geometría proyectiva, la cual se considera que tuvo su origen con el trabajo de Girard Desargues el siglo XVII. En geometría proyectiva, desaparece la noción de distancia, a pesar de que ésta es esencial en la geometría euclidiana. Además, en geometría proyectiva, todas las rectas tienen un punto de intersección. En particular, el punto de intersección de dos o más rectas paralelas es un punto al infinito, y el conjunto de todas estas rectas es la línea al infinito.

En la formulación y en la demostración de nuestro resultado, aparecen nociones propias de la geometría proyectiva, por ejemplo, el concepto de recta polar y el Teorema de Menelao.

Con el fin de hacer esta exposición más accesible, se ha intentado presentar los resultados utilizando herramientas de geometría euclidiana, con las que el lector, en mayor o menor medida, tiene alguna familiaridad. Procedemos a presentar algunas herramientas en geometría que serán de gran utilidad en este trabajo: teoría de rectas polares en el plano y potencia de un punto respecto a un círculo. 


\subsection{Rectas Polares. Construcción geométrica.}

El resultado principal presentado en este trabajo (Teorema 2.1) está dado en términos de la noción de recta polar de un punto respecto a una circunferencia. En [1, 3] se puede consultar una presentación de esta noción en términos de teoría de inversión. Otra exposición distinta se halla en [2]. Para nuestros fines, presentamos la siguiente definición.

Consideremos una circunferencia $\omega$ en el plano y un punto $P$ fuera de ella. Desde el punto $P$ se trazan las rectas tangentes a $\omega$. Llamémosles $P_{1}$ y $P_{2}$ a los puntos de tangencia de dichas rectas. A la recta $\mathscr{L}_{P}$, determinada por los puntos $P_{1}$ y $P_{2}$, le llamamos la recta polar del punto $P$ con respecto a la circunferencia $\omega$.

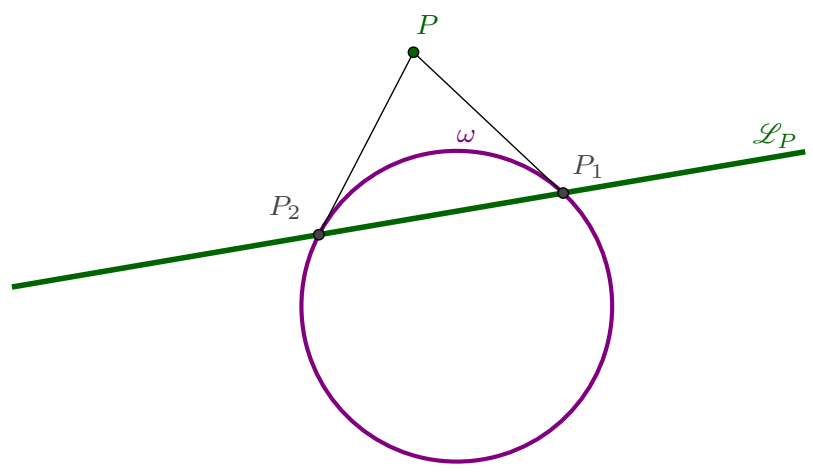

Figura 1: $\mathscr{L}_{P}$ es la recta polar de $P$ respecto de $\omega$.

Tomemos ahora un punto $Q$ sobre la circunferencia $\omega$. En este caso, la recta polar $\mathscr{L}_{Q}$ es la tangente a $\omega$ por dicho punto $Q$ :

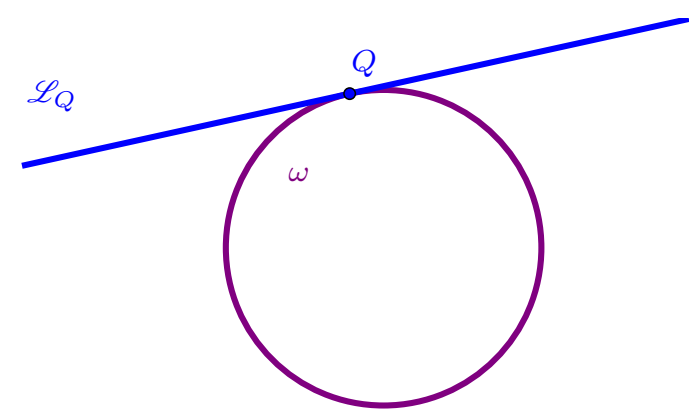

Figura 2: $\mathscr{L}_{Q}$ es la recta polar respecto de $\omega$ del punto $Q$.

Finalmente, veamos el caso cuando el punto $R$ está en el interior de $\omega$. Llamémosle $O$ al centro de la circunferencia, y por el punto $R$ tracemos la cuerda con extremos $R_{1}$ y $R_{2}$, de manera que $R_{1} R_{2}$ sea perpendicular a $O R$. Por los puntos $R_{1}$ y $R_{2}$ trazamos las tangentes a $\omega$ hasta intersecarse en el punto $R^{\prime}$. Finalmente, la recta $\mathscr{L}_{R}$, que es perpendicular a $R^{\prime} R$ y que pasa por $R^{\prime}$, le llamamos la recta polar de $R$ con respecto a $\omega$.

De esta forma, hemos definido el concepto de recta polar para (casi) todo punto del plano. La desventaja de definir las rectas polares así es que parece que en cada caso (con el punto 


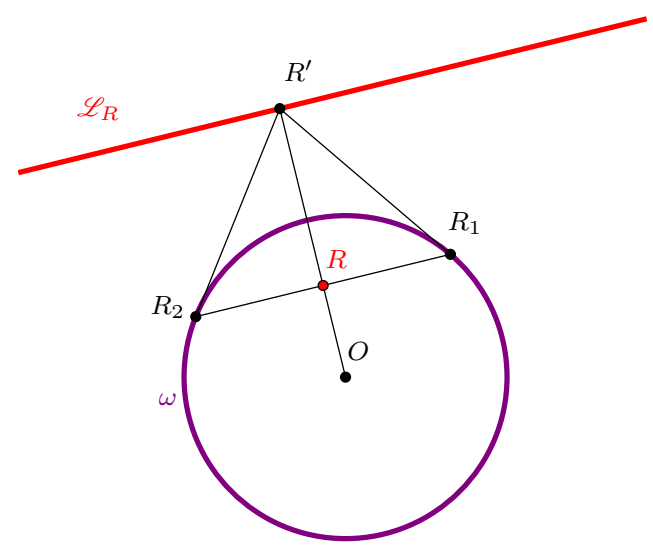

Figura 3: Una manera de construir la recta polar $\mathscr{L}_{R}$, si $R$ está en el interior de $\omega$.

dentro, fuera o sobre la circunferencia) obtenemos rectas que no guradan ninguna relación entre ellas. En [1, 3] podemos encontrar una definición unificada, pero el lector interesado en convencerse por sí mismo de que las definiciones aquí presentadas son en realidad una sola, puede hacerlo resolviendo el siguiente resultado:

Lema 1.1 Sea $\omega$ la circunferencia de centro en el origen y radio 1 . Si $P=(a, b)$ es un punto cualquiera en el plano, entonces la ecuación de la recta polar $\mathscr{L}_{P}$ es ax + by $=1$. Esto, sin importar si $P$ está dentro, sobre o fuera de $\omega$.

El software libre GeoGebra incluye una herramienta para construir la recta polar de un punto respecto a una circunferencia. Se invita al lector a descargarlo (click aquí) y verificar que la recta polar está definida para todo punto del plano, salvo el centro de la circunferencia.

Observaciones 1.1 Aunque parece ser de geometría euclidiana, el concepto de recta polar corresponde a la geometría proyectiva. Por ejemplo, las rectas polares pueden construirse usando sólo regla, y en términos de cualquier cónica, no sólo una circunferencia (ver [2]). Además, no está definida la recta polar para el centro $O$ de la circunferencia, ni existen puntos cuya recta polar pase por $O$. Sin embargo, desde el punto de vista de la geometría proyectiva, la recta polar de $O$ es la línea al infinito, y cada recta que pasa por $O$ es la recta polar de algún punto al infinito. Esto puede intuirse a partir de la definición aquí presentada.

Proposición 1.2 Sean $\omega$ una circunferencia en el plano, P, $Q$ y $R$ puntos en el mismo plano y $\mathscr{L}_{P}, \mathscr{L}_{Q}$ y $\mathscr{L}_{R}$ sus respectivas rectas polares respecto de $\omega$. Algunas propiedades interesantes de las rectas polares de puntos con respecto a una circunferencia son las siguientes:

- Si P pertenece a la recta polar $\mathscr{L}_{Q}$, entonces $Q$ pertenece a la polar $\mathscr{L}_{P}$.

- Si $R$ es la intersección de $\mathscr{L}_{P}$ y $\mathscr{L}_{Q}$, entonces $\mathscr{L}_{R}$ es precisamente $P Q$.

- Los puntos $P, Q$ y $R$ son colineales si y sólo si $\mathscr{L}_{P}, \mathscr{L}_{Q}$ y $\mathscr{L}_{R}$ concurren. 


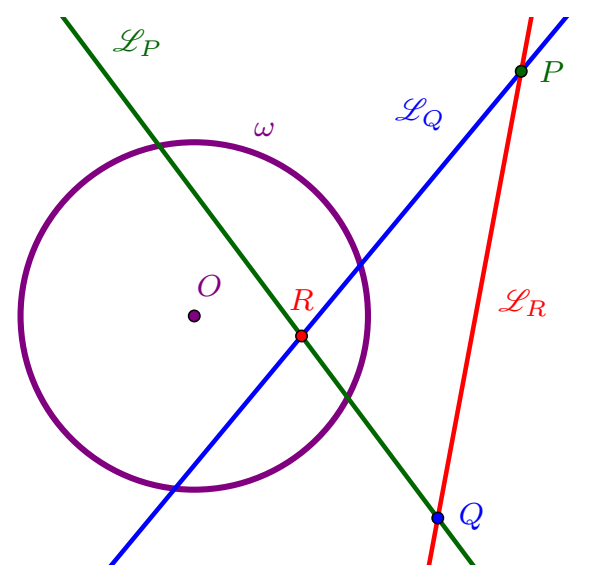

Figura 4: $\mathscr{L}_{P}$ y $\mathscr{L}_{Q}$ se intersecan en $R$. Por ello, $\mathscr{L}_{R}$ es la recta $P Q$.

La siguiente proposición nos dice que la recta polar de $P$ respecto a $\omega$ puede construirse de la siguiente forma: si trazamos dos rectas por dicho punto, de manera que corten a la circunferencia $\omega$ en cuatro puntos $A, B, C, D$, entonces los puntos $Q$ y $R$ que resultan de intersectar los lados opuestos y las diagonales de $A B C D$ están en la recta polar. Para ver una demostración de estas propiedades, puede consultar [1, 3].

Proposición 1.3 Sean $A, B, C, D$ cuatro puntos sobre una circunferencia $\omega$. Las rectas $A B$ y $C D$ se intersecan en $P$; las rectas $A D$ y $B C$ se intersecan en $Q$ y las rectas $A C$ y $B D$ se intersecan en $R$. Resulta que el triángulo $\triangle P Q R$ es autopolar, lo cual significa que

- $Q R$ es la recta polar de $P$ respecto a $\omega$, es decir, $\mathscr{L}_{P}=Q R$,

- $R P$ es la recta polar de $Q$ respecto a $\omega$, es decir, $\mathscr{L}_{Q}=R P$ y

- $P Q$ es la recta polar de $R$ respecto a $\omega$, es decir, $\mathscr{L}_{R}=P Q$.

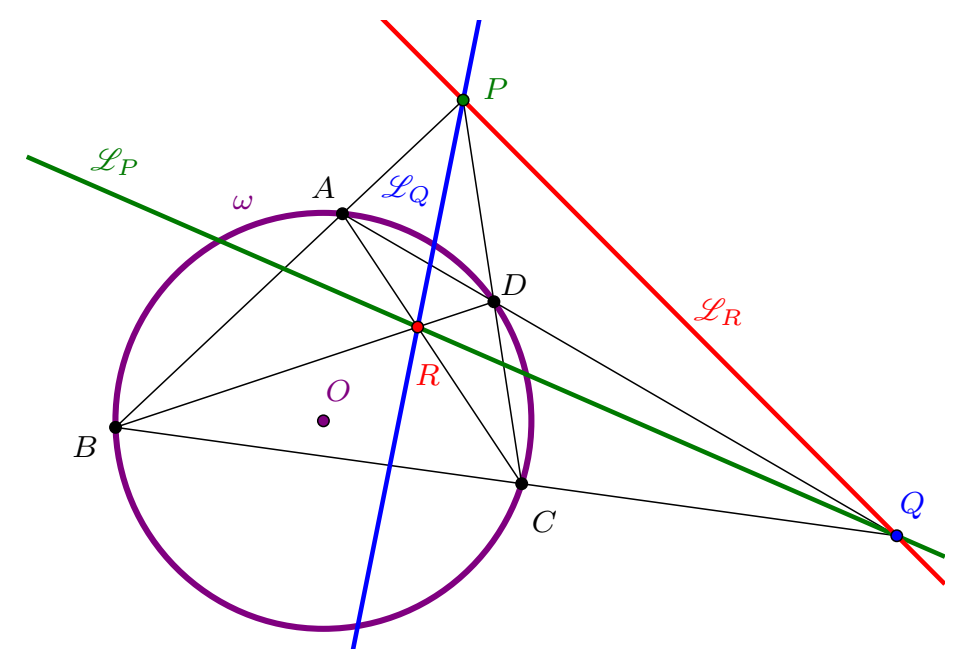

Figura 5: El triángulo $\triangle P Q R$ es autopolar. 
1.2 Potencia de un punto, ejes radicales y circunferencias ortogonales

Sea $\omega$ una circunferencia de centro $O$ y radio $R$, y sea $P$ un punto en el plano. Definimos la potencia del punto $P$ con respecto a la circunferencia $\omega$ como el número $P O^{2}-R^{2}$. En lo sucesivo, denotaremos a la potencia de $P$ respecto de $\omega$ como $\mathcal{P}_{\omega}(P)$.

Es inmediato de la definición notar que si $P$ es un punto en el exterior de la circunferencia $\omega$, entonces la potencia de $P$ respecto a $\omega$ es un número positivo. Similarmente, si $P$ está en el interior, entonces dicha potencia es negativa, y en el caso en que $P$ está sobre $\omega$, entonces la potencia de $P$ respecto a $\omega$ es igual a cero.

La potencia de un punto respecto a una circunferencia es importante debido a que en cierto sentido es un invariante geométrico. Para explicarlo, consideremos una circunferencia $\omega$ y un punto $P$ distinto de su centro $O$. Sean $X$ e $Y$ las intersecciones de la recta $P O$ con $\omega$, donde $X$ es la intersección más cercana a $P$.
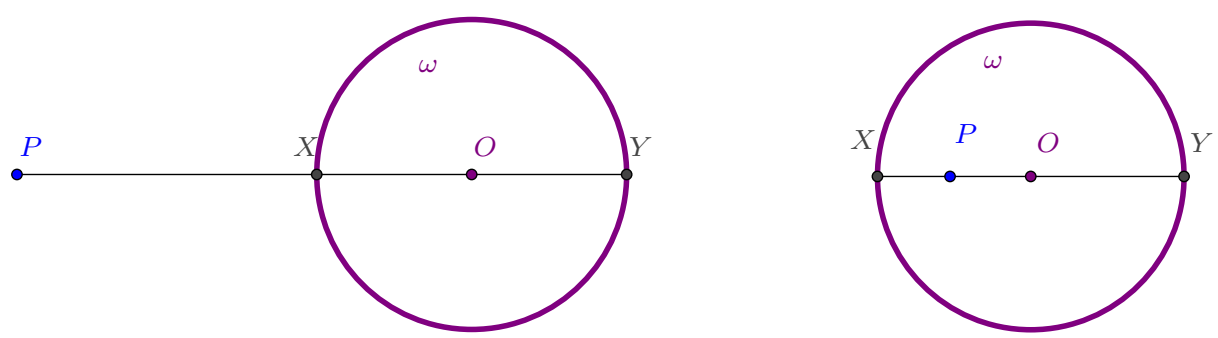

Figura 6: Una interpretación geométrica: $\mathcal{P}_{\omega}(P)=P X \cdot P Y$.

Observemos que $P X=P O-X O=P O-R$ y $P Y=P O+O Y=P O+R$ (como segmentos dirigidos), por lo que $P X \cdot P Y=P O^{2}-R^{2}=\mathcal{P}_{\omega}(P)$. Sin embargo, la fórmula $\mathcal{P}_{\omega}(P)=P X \cdot P Y$ es válida incluso para cualquier recta por $P$ que interseca a $\omega$, no sólo para la recta $P O$. En efecto, tracemos una segunda recta por $P$, y sean $A, B$ los puntos de intersección de dicha recta con $\omega$.
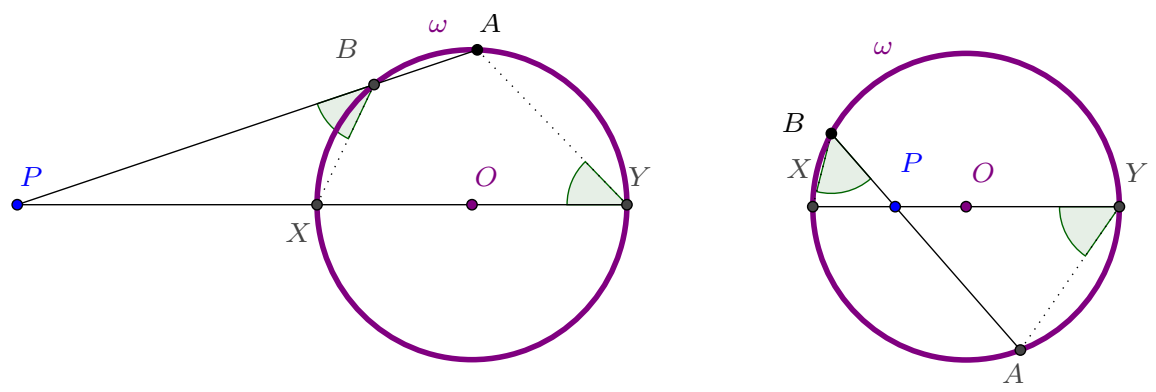

Figura 7: En los dos casos, con $P$ dentro o fuera de $\omega$, se tiene que $\mathcal{P}_{\omega}(P)=P A \cdot P B$.

En la primera figura, los ángulos $\angle P B X$ y $\angle P Y A$ son suplementarios a $\angle X B A$, mientras que en la segunda figura, los ángulos $\angle P B X$ y $\angle P Y A$ subtienden el arco $\widehat{A X}$. En cualquier caso, se tiene la igualdad de ángulos $\angle P B X=\angle P Y A$, la cual, implica la semejanza de los triángulos $\triangle P B X$ y $\triangle P Y A$. Por tanto, sus lados correspondientes son proporcionales: $\frac{P X}{P A}=\frac{P B}{P Y}$. Esta igualdad se reescribe como $P A \cdot P B=P X \cdot P Y=\mathcal{P}_{\omega}(P)$. 
Finalmente, cuando $P$ es exterior a $\omega$, trazamos una tangente por $P$ a $\omega$.

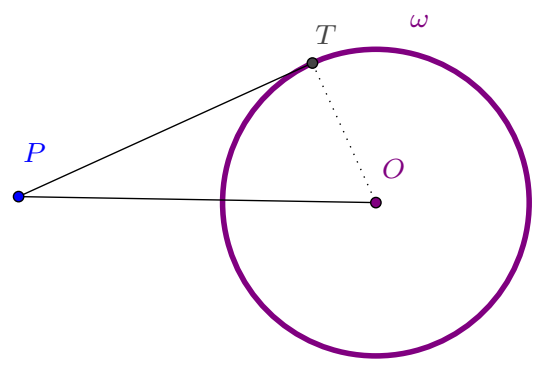

Figura 8: Para $P$ exterior a $\omega$, la longitud de la tangente $P T$ satisface que $\mathcal{P}_{\omega}(P)=P T^{2}$.

Puesto que el radio $O T$ es perpendicular a la tangente $P T$, se tiene que el triángulo $\triangle P O T$ es rectángulo. Por el Teorema de Pitágoras, $P T^{2}=P O^{2}-R^{2}=\mathcal{P}_{\omega}(P)$, por lo que, geométricamente, la potencia de $P$ es igual al cuadrado de la longitud de la tangente $P T$.

Es importante resaltar que la noción de potencia de un punto respecto a una circunferencia $\omega$ puede definirse sin ningún cambio si $\omega$ es un punto, es decir, una circunferencia de radio cero. Esta noción será de utilidad a lo largo de la Sección 2.

Sean ahora $\mathscr{C}$ y $\omega$ dos circunferencias y sea $P$ un punto en el plano. En general, la potencia de $P$ respecto a $\omega$ es un número distinto de la potencia de $P$ respecto a $\mathscr{C}$. Naturalmente, existen puntos $P$ en el plano para los cuales las dos potencias son iguales. Se puede demostrar que el lugar geométrico de los puntos $P$ cuyas potencias respecto a $\mathscr{C}$ y $\omega$ son iguales es una línea recta, conocida como eje radical de $\mathscr{C}$ y $\omega$. Cuando las circunferencias se intersecan, el eje radical pasa por los puntos de intersección, pero en general, siempre es perpendicular a la recta que une los centros de las circunferencias.
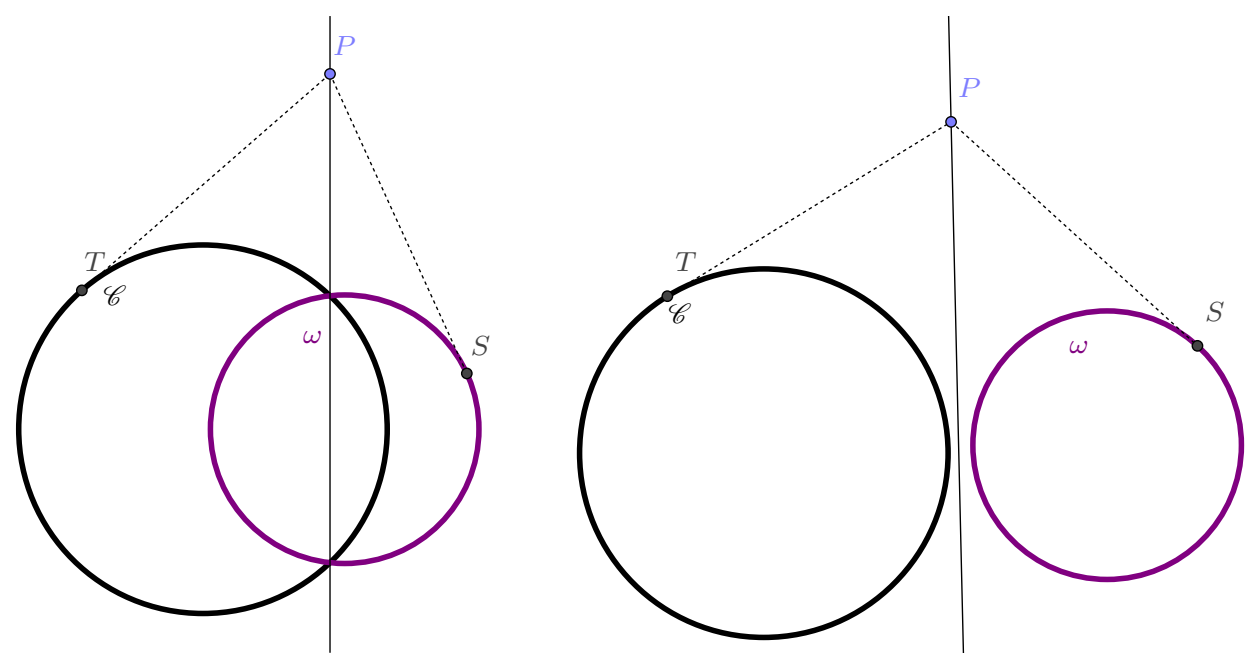

Figura 9: La recta vertical es el eje radical de $\mathscr{C}$ y $\omega$. Como $P$ pertenece al eje radical de $\mathscr{C}$ y $\omega$, las tangentes desde $P$ a $\mathscr{C}$ y $\omega$ son de igual longitud.

Para cada par de circunferencias, su eje radical es una línea recta. En algunas ocasiones, se tiene que tres o más circunferencias tienen el mismo eje radical dos a dos. En este caso, se dice que las circunferencias son coaxiales, como en la Figura 10. 


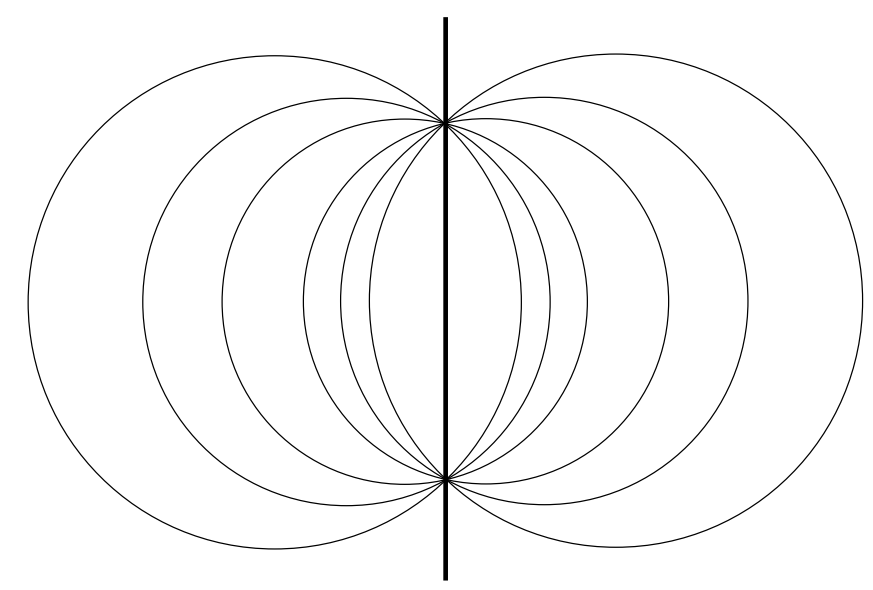

Figura 10: Una familia de circunferencias coaxiales.

Sin embargo, en general, tres circunferencias determinan tres ejes radicales, uno para cada par de circunferencias. En ese caso, los tres ejes radicales concurren en un punto llamado centro radical, el cual tiene la misma potencia con respecto a las tres circunferencias.

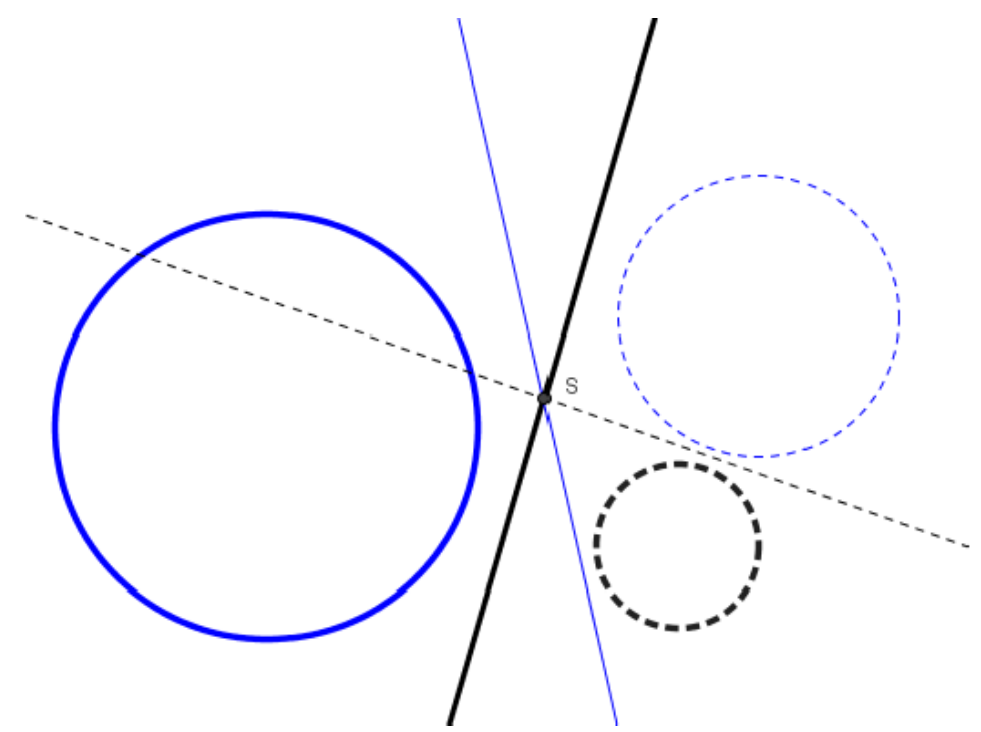

Figura 11: La recta azul es el eje radical de las circunferencias azules. La recta punteada es el eje radical de las circunferencias punteadas. La recta de trazo grueso es el eje radical de las circunferencias de trazo grueso. $S$ es el centro radical de las tres circunferencias.

Si desde el centro radical trazamos las tangentes a las tres circunferencias, dichas tangentes tendrán igual longitud, porque el cuadrado de la longitud de cada tangente es igual a la potencia común desde dicho centro. Tomando la longitud de las tangentes como radio, y el centro radical como centro, tenemos una circunferencia que pasa por los puntos de tangencia. En otras palabras, los radios de esta circunferencia son tangentes a las tres circunferencias.

En general, cuando dos circunferencias se intersecan y una de ellas tiene dos radios tangentes a la otra, se dice que ambas circunferencias son ortogonales. 


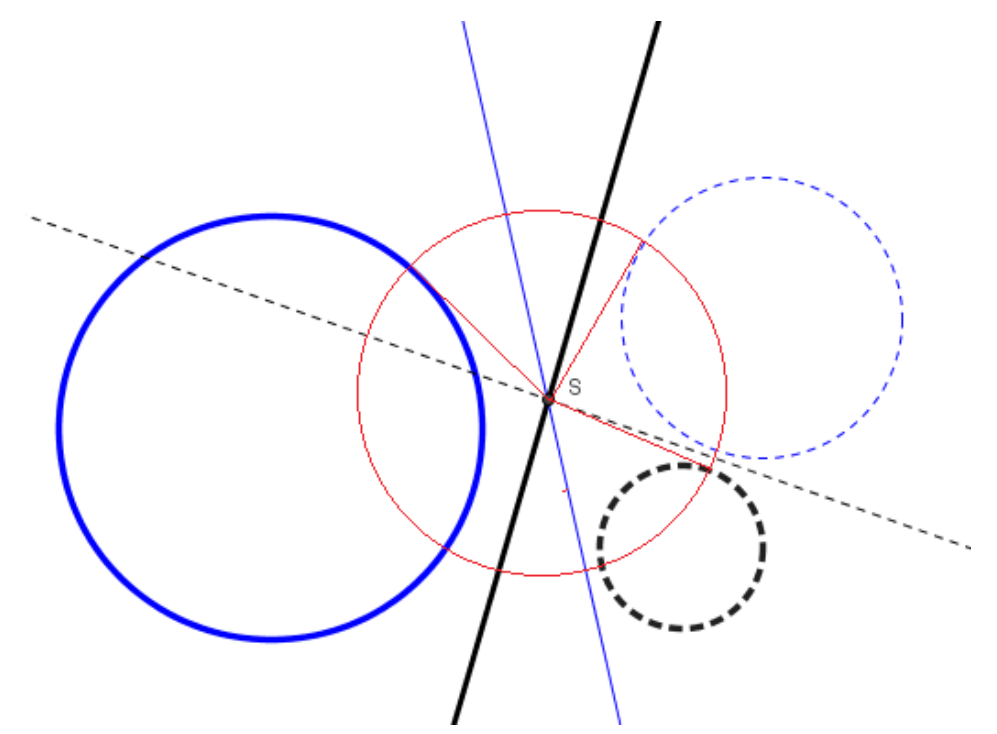

Figura 12: La circunferencia roja es ortogonal a las otras tres.

\section{Resultado principal y su demostración}

Procedemos ahora a enunciar el resultado principal de este trabajo, el cual es un criterio de colinealidad para puntos sobre los lados de un triángulo. Esta construcción utiliza la noción de recta polar de un punto respecto a una circunferencia.

Teorema 2.1 Sean $\triangle A B C$ un triángulo y $\omega$ una circunferencia en el plano. Sean $\mathscr{L}_{A}$, $\mathscr{L}_{B}$ y $\mathscr{L}_{C}$ las respectivas rectas polares de $A, B$ y $C$ con respecto a $\omega$. Sean $\widetilde{A}$ la intersección de las rectas $\mathscr{L}_{A}$ y $B C, \widetilde{B}$ la intersección de las rectas $\mathscr{L}_{B}$ y $C A$, y $\widetilde{C}$ la intersección de las rectas $\mathscr{L}_{C}$ y $A B$. Se tiene que los puntos $\widetilde{A}, \widetilde{B}, \widetilde{C}$ son colineales.

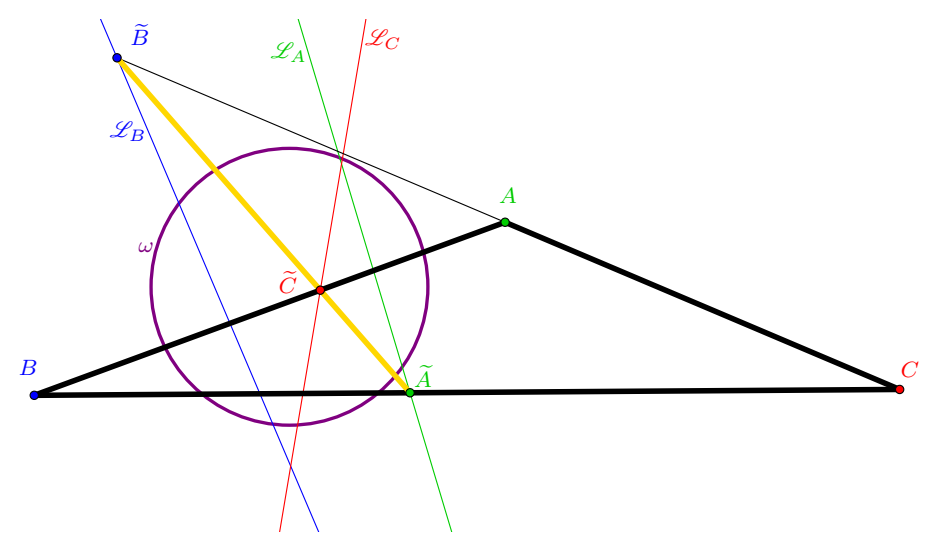

Figura 13: $\triangle A B C$ es un triángulo y $\omega$ una circunferencia. Las rectas $\mathscr{L}_{A}$ (verde), $\mathscr{L}_{B}$ (azul) y $\mathscr{L}_{C}$ (rojo) son las rectas polares de los vértices $A, B$ y $C$, respectivamente. La línea en amarillo es la determinada por los puntos colineales $\widetilde{A}, \widetilde{B}$ y $\widetilde{C}$.

Para dar la prueba del Teorema 2.1, necesitamos algunos resultados previos. En la demostración del primero de ellos, utilizaremos el Teorema de Menelao, que es el resultado 
de colinealidad por excelencia, así como el Teorema generalizado de la bisectriz. En [3] se puede consultar una demostración de ambos resultados.

Teorema 2.2 (Teorema de Menelao) Sea $\triangle A B C$ un triángulo y sean $\widetilde{A}, \widetilde{B}, \widetilde{C}$ puntos en las rectas $B C, C A, A B$, respectivamente.

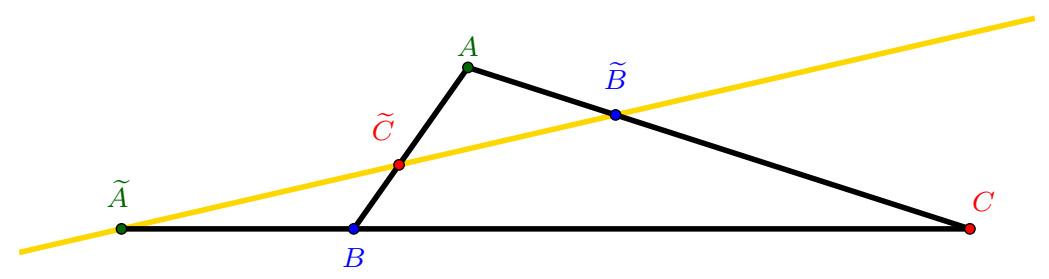

Los puntos $\widetilde{A}, \widetilde{B}, \widetilde{C}$ son colineales si y sólo si $\frac{C \widetilde{A}}{\widetilde{A} B} \cdot \frac{B \widetilde{C}}{\widetilde{C} A} \cdot \frac{A \widetilde{B}}{\widetilde{B} C}=-1$.

Este teorema corresponde al campo de la geometría proyectiva porque, estrictamente hablando, la colinealidad no depende de las longitudes de los segmentos involucrados, sino solamente de las proporciones de los mismos.

Teorema 2.3 (Teorema de la bisectriz generalizado) Sea $\triangle A B C$ un triángulo y sea $P$ un punto en la recta $B C$. Se tiene que $\frac{B P}{P C}=\frac{B A}{A C} \cdot \frac{\operatorname{sen} \angle B A P}{\operatorname{sen} \angle P A C}$.

Ahora aplicaremos los teoremas anteriores para probar lo siguiente:

Proposición 2.4 Sea $\triangle A B C$ un triángulo, y sea $O$ un punto que no pertenece a ninguna de sus alturas. Sean $\widetilde{A}$ la intersección de $B C$ con la perpendicular a $O A$ que pasa por $O, \widetilde{B}$ la intersección de $C A$ con la perpendicular a $O B$ que pasa por $O$ y $\widetilde{C}$ la intersección de $A B$ con la perpendicular a $O C$ que pasa por $O$. Se tiene que los puntos $\widetilde{A}, \widetilde{B}, \widetilde{C}$ son colineales.

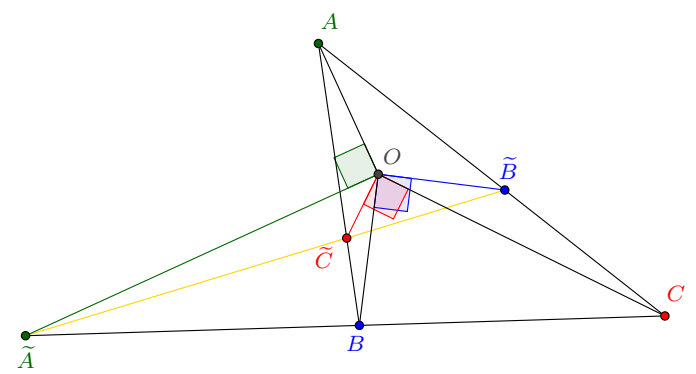

Figura 14: Ilustración de la Proposición 2.4.

Nótese que la Proposición 2.4 es un caso degenerado del Teorema 2.1 cuando la circunferencia $\omega$ es de radio cero. En efecto, si $O$ y $P$ son puntos distintos, la polar de $P$ respecto a $O$, como circunferencia de radio cero, se entiende como la perpendicular a $O P$ por $O$. 
PRUEBA. Por construcción, se tienen las siguientes igualdades de ángulos dirigidos:

$$
\angle B O \widetilde{A}=\angle \widetilde{B} O A, \quad \angle C O \widetilde{B}=\angle \widetilde{C} O B, \quad \angle A O \widetilde{C}=\angle \widetilde{A} O C .
$$

En consecuencia, $\frac{\operatorname{sen} \angle B O \widetilde{A}}{\operatorname{sen} \angle \widetilde{B} O A}=\frac{\operatorname{sen} \angle C O \widetilde{B}}{\operatorname{sen} \angle \widetilde{C O B}}=\frac{\operatorname{sen} \angle A O \widetilde{C}}{\operatorname{sen} \angle \widetilde{A} O C}=1$. Por el Teorema generalizado de la bisectriz aplicado a $\widetilde{A}$ y al triángulo $\triangle B O C$, la razón en que $\widetilde{A}$ divide al segmento $B C$ puede calcularse por $\frac{B \widetilde{A}}{\widetilde{A} C}=\frac{B O}{O C} \cdot \frac{\operatorname{sen} \angle B O \widetilde{A}}{\operatorname{sen} \angle \widetilde{A} O C}$. Similarmente, tenemos que $\frac{C \widetilde{B}}{\widetilde{B} A}=\frac{C O}{O A} \cdot \frac{\operatorname{sen} \angle C O \widetilde{B}}{\operatorname{sen} \angle \widetilde{B} O A} \mathrm{y}$ $\frac{A \widetilde{C}}{\widetilde{C} B}=\frac{A O}{O B} \cdot \frac{\operatorname{sen} \angle A O \widetilde{C}}{\operatorname{sen} \angle \widetilde{C} O B}$. Multiplicando y considerando segmentos dirigidos,

$$
\frac{B \widetilde{A}}{\widetilde{A} C} \cdot \frac{C \widetilde{B}}{\widetilde{B} A} \cdot \frac{A \widetilde{C}}{\widetilde{C} B}=\frac{B O}{O C} \cdot \frac{C O}{O A} \cdot \frac{A O}{O B} \cdot \frac{\operatorname{sen} \angle B O \widetilde{A}}{\operatorname{sen} \angle \widetilde{A} O C} \cdot \frac{\operatorname{sen} \angle C O \widetilde{B}}{\operatorname{sen} \angle \widetilde{B} O A} \cdot \frac{\operatorname{sen} \angle A O \widetilde{C}}{\operatorname{sen} \angle \widetilde{C} O B}=-1 \text {. }
$$

Por el Teorema de Menelao, los puntos $\widetilde{A}, \widetilde{B}, \widetilde{C}$ son colineales.

Teniendo en cuenta el resultado de la Proposición 2.4, es fácil convencerse de que el recíproco también es cierto. En otras palabras, dados tres puntos colineales $\widetilde{A}, \widetilde{B}, \widetilde{C}$ sobre los lados de un triángulo $\triangle A B C$, existe un punto $P$ tal que los ángulos $\angle A P \widetilde{A}, \angle B P \widetilde{B}$, $\angle C P \widetilde{C}$ son todos rectos. Más aún, como veremos en el siguiente resultado, el punto $P$ no es único, sino que existe un segundo punto $P^{\prime}$ con la misma propiedad. En lo sucesivo, denotaremos por $\mathscr{C}_{A}, \mathscr{C}_{B}, \mathscr{C}_{C}$ a las circunferencias de diámetros $\widetilde{A} A, \widetilde{B} B, \widetilde{C} C$.

Lema 2.5 Sea $\triangle A B C$ un triángulo y sean $\widetilde{A}, \widetilde{B}$ y $\widetilde{C}$ puntos colineales en las rectas $B C$, $C A$ y $A B$, respectivamente. Se tiene que las circunferencias $\mathscr{C}_{A}, \mathscr{C}_{B}, \mathscr{C}_{C}$ tienen dos puntos en común.

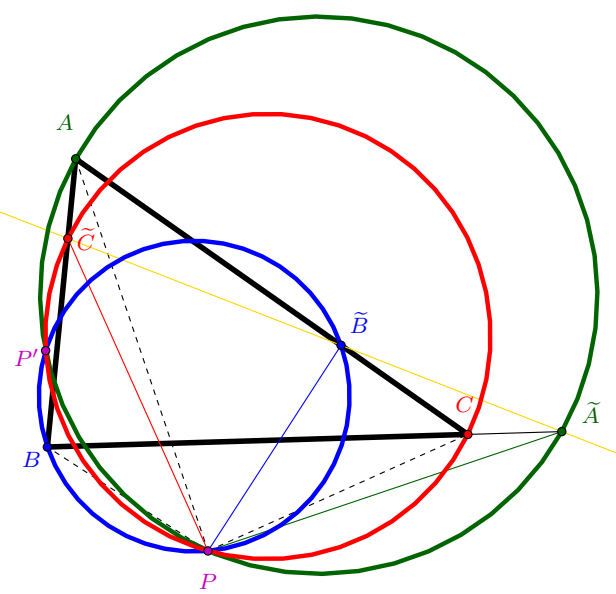

Figura 15: $P$ y $P^{\prime}$ son los puntos $O$ que dan lugar a $\widetilde{A}, \widetilde{B}$ y $\widetilde{C}$ de la Proposición 2.4.

Prueba. Sean $P$ y $P^{\prime}$ las intersecciones de las circunferencias $\mathscr{C}_{A}$ y $\mathscr{C}_{B}$ de diámetros $\widetilde{A} A$ y $\widetilde{B} B$. Por construcción, se tiene que $A P$ es perpendicular a $P \widetilde{A}$ y que $B P$ es perpendicular a $P \widetilde{B}$. Sea $\widetilde{C}_{1}$ la intersección de la perpendicular a $C P$ por $P$ con la recta $B C$. Por la 
Proposición 2.4, los puntos $\widetilde{A}, \widetilde{B}$ y $\widetilde{C}_{1}$ son colineales. En otras palabras, $\widetilde{C}_{1}$ es la intersección de las rectas $A B$ y $\widetilde{A} \widetilde{B}$. Puesto que, por hipótesis, $\widetilde{C}$ es la intersección de las rectas $A B$ y $\widetilde{A} \widetilde{B}$, tenemos que $\widetilde{C}_{1}=\widetilde{C}$. En particular, $P$ pertenece a la circunferencia de diámetro $\widetilde{C} C$. De manera similar, $P^{\prime}$ pertenece a la circunferencia de diámetro $\widetilde{C} C$.

El siguiente resultado es el último que necesitamos para demostrar el Teorema 2.1, el cual probaremos usando geometría euclidiana. Existe una demostración más inmediata usando teoría de inversión. Se invita al lector a realizar dicha demostración.

Lema 2.6 Sean $A, \widetilde{A}$ puntos distintos y $\omega$ una circunferencia. La recta polar $\mathscr{L}_{A}$ del punto $A$ respecto a $\omega$ pasa por $\widetilde{A}$ si y sólo si la circunferencia $\mathscr{C}_{A}$ de diámetro $A \widetilde{A}$ y $\omega$ son ortogonales.

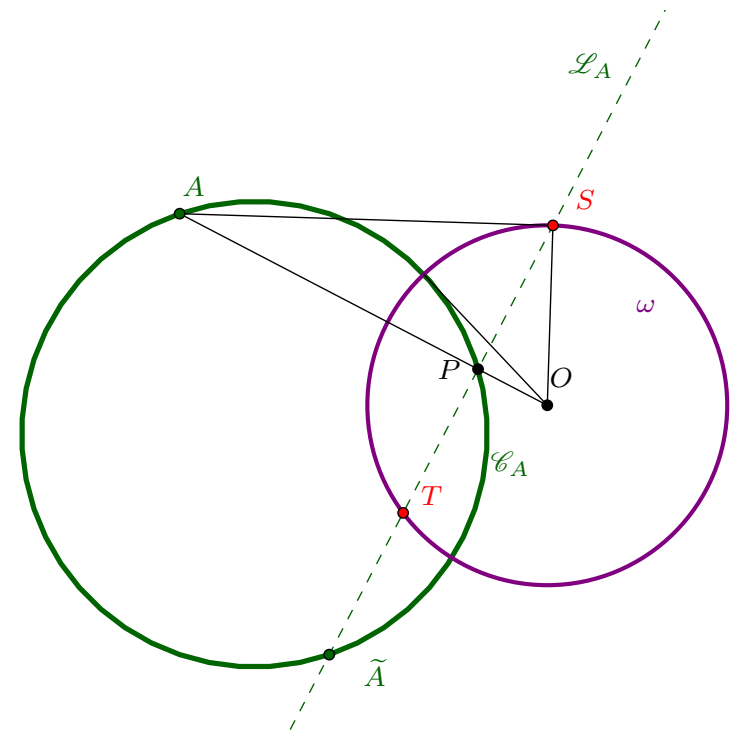

Prueba. Si $A$ está sobre $\omega$, el resultado es inmediato de la definición de recta polar para este caso. Sin pérdida de generalidad, puede suponerse que $A$ está fuera de la circunferencia, de lo contrario se puede intercambiar el papel de $\widetilde{A}$ y $A$ (¿por qué?). Sean $O$ el centro de $\omega, P$ la segunda intersección de $\mathscr{C}_{A}$ con la recta $A O$, y $S, T$ las intersecciones de $\widetilde{A} P$ con $\omega$. Por ser $A \widetilde{A}$ el diámetro de $\mathscr{C}_{A}$, se tiene que $\angle \widetilde{A} P A=90^{\circ}$, es decir, $\widetilde{A} P$ es perpendicular a $O A$. Puesto que la polar $\mathscr{L}_{A}$ es perpendicular a $O A$, se tiene que $\mathscr{L}_{A}$ pasa por $\widetilde{A}$ si y sólo si $\widetilde{A} P$ es la polar de $\omega$. Por definición de $\mathscr{L}_{A}$, esto último ocurre si y sólo si $S$ y $T$ son los puntos donde las tangentes desde $A$ tocan a $\omega$. A su vez, lo anterior se tiene si y sólo si $\angle A S O=90^{\circ}$. Como $\angle S P O$ es recto, se tiene que $\angle A S O=90^{\circ}$ si y sólo si los triángulos $\triangle A S O$ y $\triangle S P O$ son semejantes. Esto ocurre si y sólo si $\frac{O S}{O A}=\frac{O P}{O S}$, es decir, si y sólo si $O A \cdot O P=R^{2}$, donde $R$ es el radio de $\omega$. Puesto que $O A \cdot O P$ es la potencia de $O$ respecto de $\mathscr{C}_{A}$, se tiene que $R^{2}$ es igual al cuadrado de la longitud de la tangente desde $O$ a $\mathscr{C}_{A}$. Esto es equivalente a que $\omega$ sea ortogonal a $\mathscr{C}_{A}$, como queríamos.

Con todos estos resultados en nuestras manos, procedamos a probar el Teorema 2.1. 
PruebA. Por hipótesis, la polar de $A$ respecto a $\omega$ pasa por $\widetilde{A}$. Por el Lema 2.6, se concluye que las circunferencias $\mathscr{C}_{A}$ y $\omega$ son ortogonales. Similarmente, $\mathscr{C}_{B}$ y $\omega$ también lo son. Sean $P$ y $P^{\prime}$ los puntos de intersección de $\mathscr{C}_{A}$ con $\mathscr{C}_{B}$. Sea $\widetilde{C}_{0}$ el punto de intersección de la recta $\widetilde{A} \widetilde{B}$ con $A B$. Basta entonces con demostrar que $\widetilde{C}_{0}=\widetilde{C}$. Por ser $\widetilde{A}, \widetilde{B}, \widetilde{C}_{0}$ colineales, el Lema 2.5 implica que la circunferencia $\mathscr{C}_{0}$ de diámetro $C \widetilde{C}_{0}$ pasa por $P$ y $P^{\prime}$. Por otra parte, como $\omega$ es ortogonal a $\mathscr{C}_{A}$ y $\mathscr{C}_{B}$, se tiene que $\omega$ es ortogonal a cualquier circunferencia que pasa por $P$ y $P^{\prime}$. En particular, $\omega$ es ortogonal a $\mathscr{C}_{0}$. Por el Lema 2.6, la polar de $C$ respecto a $\omega$ pasa por el punto en $\mathscr{C}_{0}$ diametralmente opuesto a $C$, es decir, pasa por $\widetilde{C}_{0}$. Esto significa que $\widetilde{C}_{0}$ es la intersección de $A B$ con la polar de $C$ respecto a $\omega$, es decir, $\widetilde{C}_{0}=\widetilde{C}$, como queríamos.

Observaciones 2.1 Los resultados preliminares de esta demostración serán de utilidad en la siguiente sección. Sin embargo, se puede dar una demostración directa del Teorema 2.1. por geometría analítica. Se invita al lector a llevarla a cabo como sigue:

1. Elija un sistema coordenado cuyo origen sea el centro de $\omega$ y tal que el radio de $\omega$ sea 1. Denote por $\left(x_{A}, y_{A}\right),\left(x_{B}, y_{B}\right),\left(x_{C}, y_{C}\right)$ a las coordenadas de $A, B, C$ respectivamente.

2. Utilice el Lema 1.1 y calcule las distancias dirigidas $\mathrm{d}\left(B, \mathscr{L}_{A}\right)$ y $\mathrm{d}\left(B, \mathscr{L}_{A}\right)$ de los puntos $B$ y $C$ a la recta $\mathscr{L}_{A}$. Note además que $\frac{B \widetilde{A}}{\widetilde{A} C}=-\frac{\mathrm{d}\left(B, \mathscr{L}_{A}\right)}{\mathrm{d}\left(C, \mathscr{L}_{A}\right)}$.

3. Concluya asi que $\frac{B \widetilde{A}}{\widetilde{A} C}=-\frac{x_{A} x_{B}+y_{A} y_{B}-1}{x_{A} x_{C}+y_{A} y_{C}-1}$. Obtenga expresiones similares para $\frac{C \widetilde{B}}{\widetilde{B} A}$ y $\frac{A \widetilde{C}}{\widetilde{C} B}$

4. Utilice el Teorema de Menelao para concluir.

El Teorema 2.1 es bastante general, de hecho, es válido para cualquier posición relativa entre el triángulo y la circunferencia.

Uno de los casos no comprendidos por nuestra demostración es aquél en el que exactamente una de las rectas polares es paralela al lado opuesto. Por ejemplo, si $\mathscr{L}_{A}$ y $B C$ son paralelas, entonces el punto $\widetilde{A}$ es un punto al infinito. Para que el Teorema 2.1 siga siendo válido en este caso, debe cumplirse que $\widetilde{B} \widetilde{C}$ y $B C$ son paralelas. Esto puede verificarse utilizando el punto 3 de las Observaciones 2.1 para calcular las razones $\frac{A \widetilde{B}}{\widetilde{B} C}, \frac{A \widetilde{B}}{\widetilde{B} C}$ y aplicando el Teorema de Thales. Nótese que este caso ocurre si y sólo si el centro $O$ de $\omega$ está sobre la altura del triángulo $\triangle A B C$ trazada desde $A$.

Otro de los casos ocurre si dos de las rectas polares son paralelas a los lados opuestos, digamos $\mathscr{L}_{B} \| A C$ y $\mathscr{L}_{C} \| A B$. En este caso, el centro $O$ de $\omega$ pertenece a las alturas desde $B$ y $C$. Esto significa que $O$ es el ortocentro de $\triangle A B C$, así que $O$ pertenece a la altura desde $A$. Por ello, $\mathscr{L}_{A} \| B C$. Esto significa que $\widetilde{A}, \widetilde{B}, \widetilde{C}$ son puntos al infinito, es decir, pertenecen a la línea al infinito y son colineales.

Finalmente, si alguna de las rectas polares coincide con el lado opuesto del triángulo, entonces la conclusión del Teorema 2.1 es trivial.

Con esto, hemos incluido ya todos los posibles casos en la relación del triángulo $\triangle A B C$ con la circunferencia $\omega$. Por ello, podemos concluir que el Teorema 2.1, más que un resultado de geometría euclidiana, es un resultado de geometría proyectiva. 


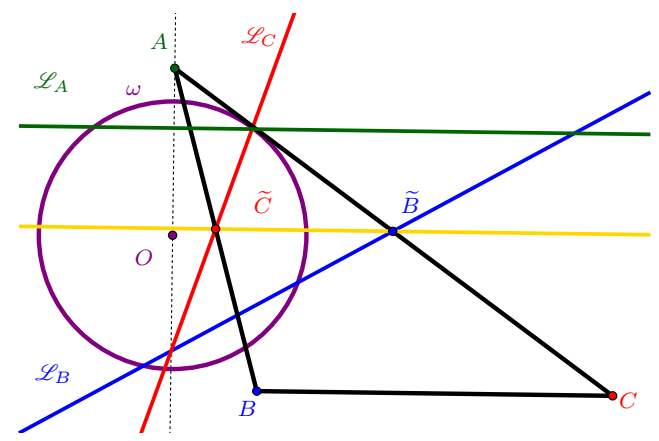

Figura 16: Cuando el centro $O$ de $\omega$ pertenece a la altura desde $A$, las rectas $\mathscr{L}_{A}$ (verde), $\widetilde{B C}$ (amarillo) y $B C$ (negro) son paralelas.

\subsection{Familias de circunferencias generadoras}

Sean $\triangle A B C$ un triángulo y $\mathscr{L}$ una recta en el plano. Es natural preguntarse si existirá alguna circunferencia $\omega$ tal que la recta $\mathscr{L}$ sea precisamente la determinada por los puntos $\widetilde{A}, \widetilde{B}, \widetilde{C}$, en el sentido del Teorema 2.1 .

Definición 2.1 Sea $\triangle A B C$ un triángulo y sea $\mathscr{L}$ una recta en el plano. Decimos que la circunferencia $\omega$ genera a $\mathscr{L}$, si $\mathscr{L}$ es la recta determinada por los puntos $\widetilde{A}, \widetilde{B}, \widetilde{C}$, en el sentido del Teorema 2.1.

En esta parte veremos que, dados un triángulo y una recta $\mathscr{L}$, la familia de circunferencias $\omega$ que generan a $\mathscr{L}$ es una familia de circunferencias coaxiales. Para demostrarlo, necesitamos el siguiente resultado.

Lema 2.7 Sean $\mathscr{C}_{1}$ y $\mathscr{C}_{2}$ circunferencias de radios $r_{1}$ y $r_{2}$, respectivamente, que se intersecan en $P$ y $P^{\prime}$. La circunferencia $\omega$ de centro $O$ y radio $R$ es ortogonal a $\mathscr{C}_{1}$ y $\mathscr{C}_{2}$ si y sólo si $\omega$ es coaxial con las circunferencias $P$ y $P^{\prime}$ de radio cero. En particular, $O$ pertenece a $P P^{\prime}$.

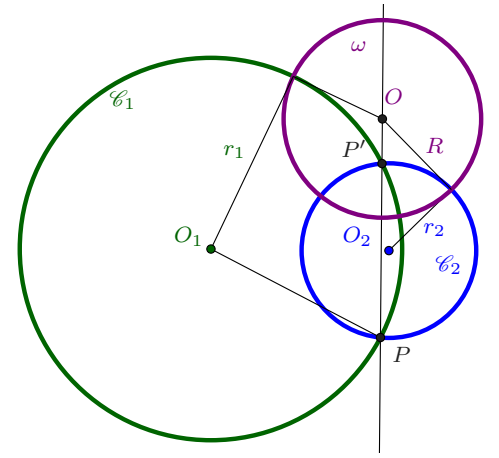

Figura 17: $\omega$ es ortogonal a $\mathscr{C}_{1}$ y $\mathscr{C}_{2}$ si y sólo si $\omega$ es coaxial con $P$ y $P^{\prime}$.

Prueba. Notemos que la mediatriz de $P P^{\prime}$ es el eje radical de $P$ y $P^{\prime}$, es decir, es la línea $O_{1} O_{2}$ de los centros de $\mathscr{C}_{1}$ y $\mathscr{C}_{2}$. De hecho, la potencia de $O_{1}$ respecto a $P$ es $r_{1}^{2}$. Más 
aún, la potencia de $O_{1}$ respecto a $\omega$ es también $r_{1}^{2}$ porque $\mathscr{C}_{1}$ es ortogonal a $\omega$. Por ello, $O_{1}$ pertenece al eje radical de $P$ y $\omega$. Análogamente, $O_{2}$ pertenece al eje radical de $P$ y $\omega$. Esto prueba que $\mathrm{O}_{1} \mathrm{O}_{2}$ es el eje radical de $P, P^{\prime}$ y $\omega$, por lo que son coaxiales. Recíprocamente, si $\omega, P$ y $P^{\prime}$ son coaxiales, entonces cada punto $Q$ de la mediatriz de $P P^{\prime}$ tiene potencia $Q P^{2}$ con respecto a $\omega$. En particular, para $O_{1}$ se tiene que, $O_{1} O^{2}-R^{2}=O_{1} P^{2}=r_{1}^{2}$, lo cual demuestra que $\omega$ y $\mathscr{C}_{1}$ son ortogonales. Análogamente, $\omega$ y $\mathscr{C}_{2}$ son ortogonales.

Teorema 2.8 Sea $\triangle A B C$ un tríangulo, y sea $\mathscr{L}$ una recta. Sean $\widetilde{A}, \widetilde{B}, \widetilde{C}$ los puntos de intersección de $\mathscr{L}$ con $B C, C A, A B$, respectivamente. Sean $P$ y $P^{\prime}$ los puntos en común de las tres circunferencias $\mathscr{C}_{A}, \mathscr{C}_{B}, \mathscr{C}_{C}$ de diámetros $A \widetilde{A}, B \widetilde{B}$ y $C \widetilde{C}$. La familia de circunferencias que generan a $\mathscr{L}$ consiste de aquéllas que son ortogonales a $\mathscr{C}_{A}, \mathscr{C}_{B}$ y $\mathscr{C}_{C}$. Equivalentemente, esta familia consiste de las circunferencias con centro en la recta $P P^{\prime}$ que son coaxiales con $P$ y $P^{\prime}$, como circunferencias de radio cero.

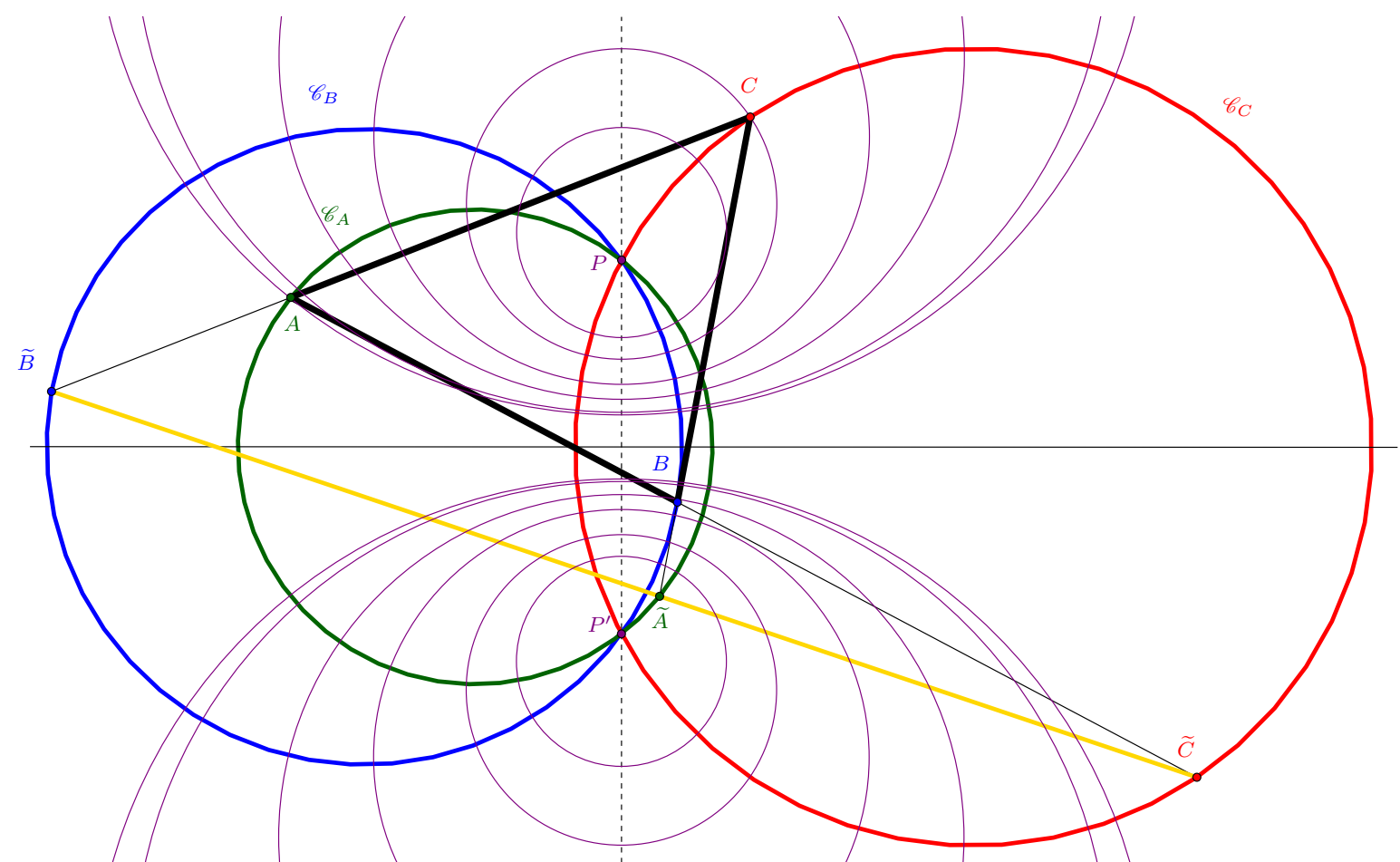

Figura 18: Para el triángulo $\triangle A B C$ y la recta $\mathscr{L}$ (amarillo), cada circunferencia en morado genera a $\mathscr{L}$. Dichas circunferencias son ortogonales a $\mathscr{C}_{A}$ (verde), $\mathscr{C}_{B}$ (azul) y $\mathscr{C}_{C}$ (roja), quienes tienen a $P$ y $P^{\prime}$ en común. La mediatriz de $P P^{\prime}$ es eje radical común de la familia.

Prueba. Supongamos que $\omega$ genera a la recta $\mathscr{L}$. Entonces, la recta polar $\mathscr{L}_{A}$ pasa por $\widetilde{A}$. Por el Lema 2.6, se tiene que $\omega$ es ortogonal a $\mathscr{C}_{A}$. Similarmente, $\omega$ es ortogonal a $\mathscr{C}_{B}$ y $\mathscr{C}_{C}$. Recíprocamente, si $\mathscr{C}_{A}, \mathscr{C}_{B}, \mathscr{C}_{C}$ son ortogonales a $\omega$, entonces, por el Lema 2.6, tenemos que $\mathscr{L}_{A}$ pasa por $\widetilde{A}, \mathscr{L}_{B}$ pasa por $\widetilde{B}, \mathscr{L}_{C}$ pasa por $\widetilde{C}$, es decir, $\omega$ genera a $\mathscr{L}$. Para probar la segunda parte, notemos que $\omega$ es ortogonal a $\mathscr{C}_{A}, \mathscr{C}_{B}$ y $\mathscr{C}_{C}$ si y sólo si es ortogonal $\mathscr{C}_{A}$ y $\mathscr{C}_{B}$, 
ya que las tres circunferencias tienen dos puntos en común. Por el Lema 2.7, esto equivale a que $\omega$ sea coaxial con $P$ y $P^{\prime}$.

\section{Ejemplos}

En esta sección, para un mismo triángulo $\triangle A B C$, aplicaremos el Teorema 2.1 para algunas circunferencias especiales $\omega$. Por ejemplo, cuando la circunferencia $\omega$ es el circuncírculo o el incírculo del triángulo, el Teorema 2.1 da lugar a resultados ya bien estudiados en geometría.

En efecto, si $\omega$ es el circuncírculo de $\triangle A B C$, entonces las rectas polares de $A, B, C$ son las tangentes a $\omega$ por dichos puntos. Esto significa que $\widetilde{A}, \widetilde{B}, \widetilde{C}$ son las intersecciones de las tangentes al circuncírculo con los lados opuestos.

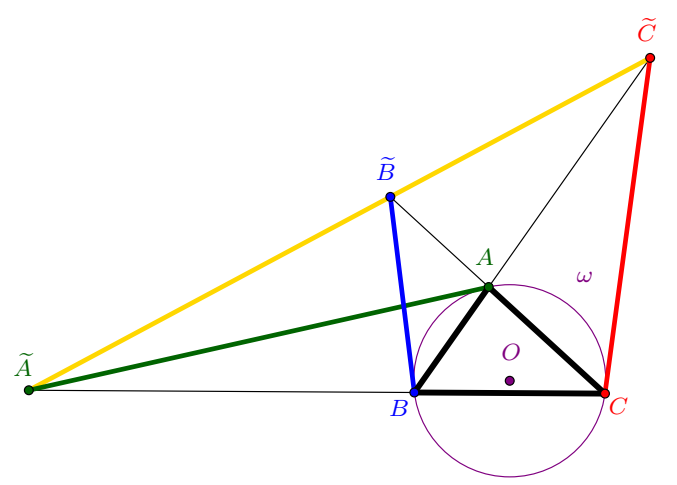

Figura 19: Si $\omega$ pasa por todos los vértices del triángulo, las rectas polares son tangentes.

Sea $\omega$ ahora el incírculo de $\triangle A B C$, y sean $D, E, F$ los puntos de tangencia de $\omega$ en los lados de $\triangle A B C$. En este caso, las rectas polares de los vértices respecto a $\omega$ son las determinadas por los puntos de tangencia.

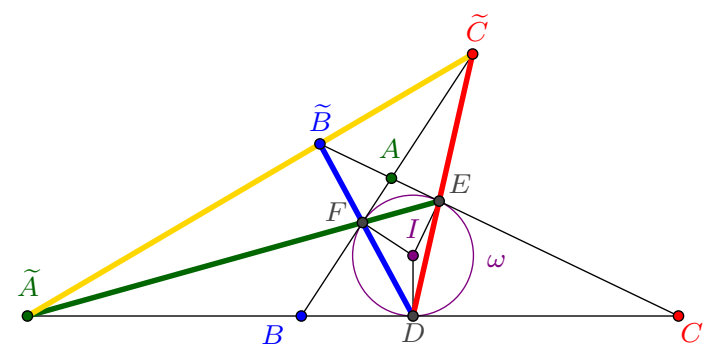

Figura 20: Este caso es como el anterior, pero aplicado al triángulo $\triangle D E F$.

Finalmente, sea $\triangle A B C$ un triángulo, y tomemos como $\omega$ a la circunferencia de diámetro $B C$. Para esta elección de $\omega$, se tiene que $\mathscr{L}_{B}$ y $\mathscr{L}_{C}$ son las perpendiculares por $B$ y $C$ al lado $B C$. Pero más interesante es estudiar la polar $\mathscr{L}_{A}$. Por construcción, el centro de $\omega$ es el punto medio $M$ de $B C$. Así, $\mathscr{L}_{A}$ es perpendicular a $A M$. Más aún, veamos que $\mathscr{L}_{A}$ pasa por el ortocentro de $\triangle A B C$. 
Sean $D$ y $E$ las intersecciones de $\omega$ con las rectas $C A$ y $A B$, respectivamente. Por ser $B C$ un diámetro de $\omega$, los ángulos $\angle B E C$ y $B D C$ son ambos rectos. Esto quiere decir que $B D$ y $C E$ son las alturas desde $B$ y $C$, respectivamente, y su punto de intersección $H$ es el ortocentro de $\triangle A B C$. Por la Proposición 1.3, las rectas $B C$ y $D E$, así como las rectas $B D$ y $C E$, se intersecan en dos puntos, los cuales pertenecen a la recta polar de $A$. El primero de ellos es entonces $\widetilde{A}$, y el segundo de ellos es $H$.

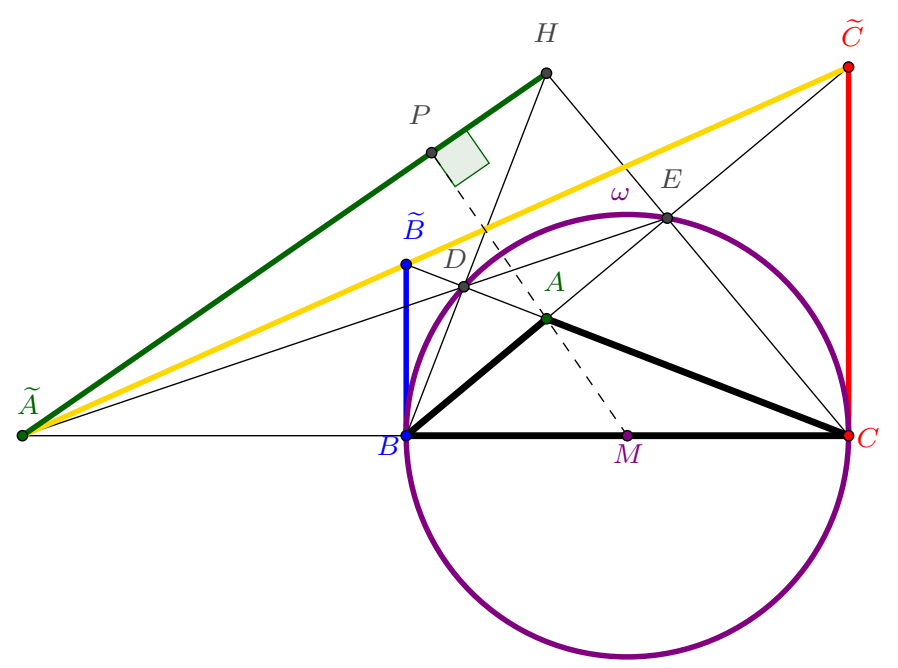

Figura 21: El caso cuando $\omega$ es de diámetro $B C$.

Esta discusión se resume en este resultado:

Proposición 3.1 Sean $\triangle A B C$ un triángulo de ortocentro $H, M$ el punto medio de $B C y$ $D$ y $E$ los pies de las alturas desde $B$ y $C$, respectivamente. Sea $\widetilde{B}$ la intersección de $C A$ con la perpendicular a $B C$ por $B$ y sea $\widetilde{C}$ la intersección de $A B$ con la perpendicular a $B C$ por $C$. Entonces, las rectas $\widetilde{B} \widetilde{C}, D E, B C$ y la perpendicular a $A M$ por $H$ son concurrentes.

\section{La Familia del Circuncírculo}

Hemos estudiado diferentes casos particulares para la circunferencia $\omega$. Por ejemplo, cuando tomamos $\omega$ como el circuncírculo del triángulo $\triangle A B C$, las polares de los vértices son las tangentes a $\omega$ pasando por cada uno de ellos. Así, la recta $\mathscr{L}$ generada en el sentido de la Definición 2.1 es la que pasa por los puntos de intersección de las tangentes al circuncírculo en cada vértice con el respectivo lado opuesto (ver Sección 3 ).

En un triángulo $\triangle A B C$, existe una circunferencia especial, llamada circunferencia de Feuerbach. También es conocida como la circunferencia de los nueve puntos porque pasa por nueve puntos notables en el triángulo, a saber:

- los puntos medios de los tres lados del triángulo,

- los pies de cada una de las tres alturas, 
- los puntos medios de los tres segmentos que unen al ortocentro con cada vértice.

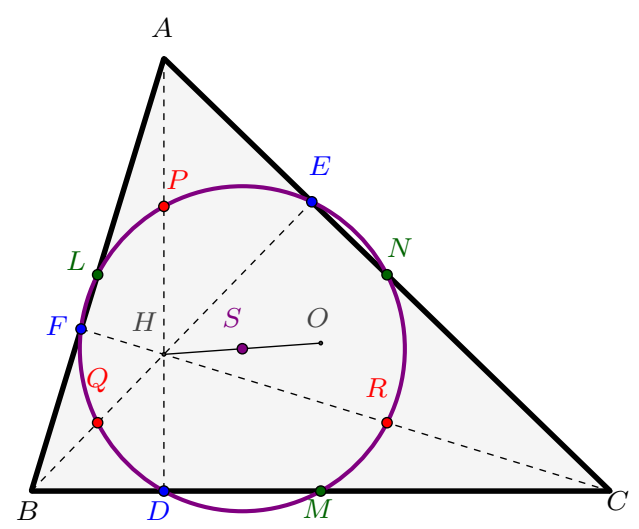

Figura 22: El centro $S$ de la circunferencia de Feuerbach es el punto medio del segmento $O H$, donde $O$ es el circuncentro del triángulo, y $H$ es su ortocentro.

Para consultar muchas de las bonitas propiedades que tiene esta circunferencia, ver [3].

Ahora estudiaremos la recta generada por la circunferencia de los nueve puntos del triángulo $\triangle A B C$. Resulta que dicha recta coincide con la recta $\mathscr{L}$ generada por el circuncírculo (Proposición 4.1). En otras palabras, ambas circunferencias pertenecen a la misma familia, en el sentido del Teorema 2.8 .

Además, apoyándonos en el Teorema 2.8, construiremos el eje radical del circuncírculo y la circunferencia de los nueve puntos, así como otras circunferencias coaxiales con las mismas. Sus centros pertenecen a una recta, conocida como la recta de Euler.

Proposición 4.1 Dado un triángulo $\triangle A B C$, su circuncírculo y su circunferencia de los nueve puntos generan a la misma recta.

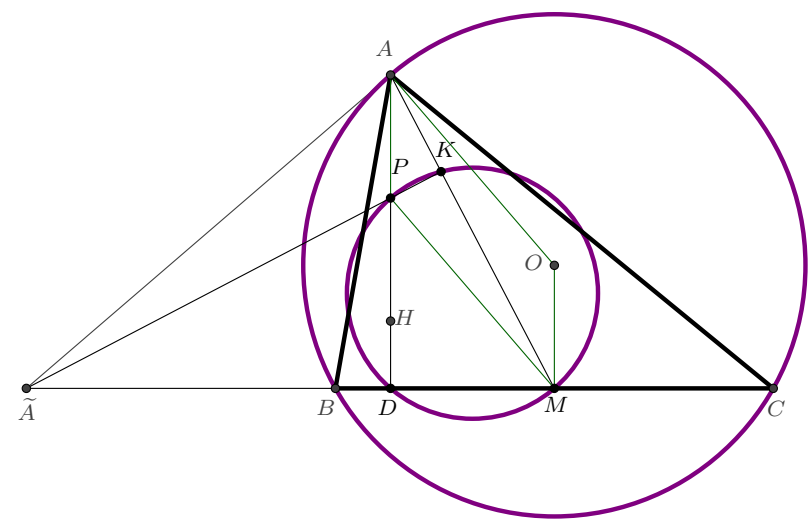

Figura 23: $\square A O M P$ es paralelogramo y $\square P K M D$ es cíclico. 
PruebA. Sean $O$ el circuncentro de $\triangle A B C, \widetilde{A}$ el punto de intersección de la tangente al circuncírculo por $A$ con $B C, D$ el pie de la altura desde $A, H$ el ortocentro, $M$ el punto medio de $B C, P$ el punto medio de $A H$ y $K$ el pie de la perpendicular de $P$ sobre $A M$.

Es un resultado conocido de geometría que $A H=2 O M$. Por ser $P$ el punto medio de $A H$, tenemos que los segmentos $A P$ y $O M$ son paralelos y de la misma longitud. Por ello, $\square A O M P$ es un paralelogramo. En particular, $M P$ y $A O$ son paralelos. Por ser $O A$ un radio, y $\widetilde{A} A$ la recta tangente del circuncírculo, se tiene que $O A$ y $\widetilde{A} A$ son perpendiculares. Por lo anterior, se tiene que $M P$ es perpendicular a $\widetilde{A} A$. Este hecho, y la perpendicularidad entre $A P$ y $M \widetilde{A}$, nos llevan a que $P$ es el ortocentro del triángulo $\triangle \widetilde{A} A M$. Por ello, los puntos $\widetilde{A}, P, K$ son colineales.

Por otro lado, $M, D, P$ pertenecen la circunferencia de los nueve puntos. Además, $\angle M D P$ es recto, por lo cual, $M P$ es diámetro de la circunferencia. Por ser $\angle P K M=90^{\circ}$, se tiene en virtud de lo anterior que $K$ pertenece también a la circunferencia de los nueve puntos del triángulo. En otras palabras, $\square P K M D$ es cíclico, pues la circunferencia de los nueve puntos pasa por todos sus vértices. Por la Proposición 1.3 , la recta polar de $A$ respecto a la circunferencia de los nueve puntos pasa por el punto de intersección de $K P$ y $M D$. En otras palabras, $\widetilde{A}$ pertenece a la polar de $A$ respecto a la circunferencia de los nueve puntos.

De manera similar se demuestra que si $\widetilde{B}$ y $\widetilde{C}$ son las intersecciones de las tangentes por $B$ y $C$ al circuncírculo con las rectas $C A$ y $A B$, entonces las rectas poalres de $B$ y $C$ respecto de la circunferencia de los nueve puntos pasan por $\widetilde{B}$ y $\widetilde{C}$. Esto significa que la recta generada por el circuncírculo es la misma que la generada por la circunferencia de los nueve puntos, en el sentido del Teorema 2.1.

\subsection{El Eje radical de la familia y la recta de Euler}

La Proposición 4.1 nos dice que la circunferencia de los nueve puntos y el circuncírculo generan a la misma recta. Por otra parte, el Teorema 2.8 asegura que la familia de circunferencias $\omega$ que generan a una misma recta tienen un mismo eje radical. Presentemos entonces algunos puntos notables en el eje radical de la circunferencia de los nueve puntos y el circuncírculo.

Sea $\widetilde{A}$ la intersección de $B C$ con la tangente por $A$ al circuncírculo de $\triangle A B C$. Similarmente, se definen $\widetilde{B}$ y $\widetilde{C}$. Sean $A^{\prime}, B^{\prime}, C^{\prime}$ los puntos medios de los segmentos $A \widetilde{A}, B \widetilde{B}, C \widetilde{C}$, es decir, los centros de las circunferencias $\mathscr{C}_{A}, \mathscr{C}_{B}, \mathscr{C}_{C}$ de diámetros $A \widetilde{A}, B \widetilde{B}, C \widetilde{C}$. Puesto que el circuncírculo y la circunferencia de los nueve puntos generan a la recta por $\widetilde{A}, \widetilde{B}$ y $\widetilde{C}$, el Lema 2.7 nos dice que $\mathscr{C}_{A}, \mathscr{C}_{B}$ y $\mathscr{C}_{C}$ son ortogonales al circuncírculo y la circunferencia de los nueve puntos. Además, los centros $A^{\prime}, B^{\prime}$ y $C^{\prime}$ de $\mathscr{C}_{A}, \mathscr{C}_{B}$ y $\mathscr{C}_{C}$ están sobre el eje radical de esas dos circunferencias. Por otro lado, sean $D, E, F$ los pies de las alturas, y $A_{1}, B_{1}, C_{1}$ las intersecciones de los pares de rectas: $E F$ con $B C, F D$ con $C A$, y $D E$ con $A B$. Es posible demostrar también que $A_{1}, B_{1}$ y $C_{1}$ están sobre el eje radical de ambas circunferencias.

Otro elemento importante para construir a las circunferencias de la familia del cir- 


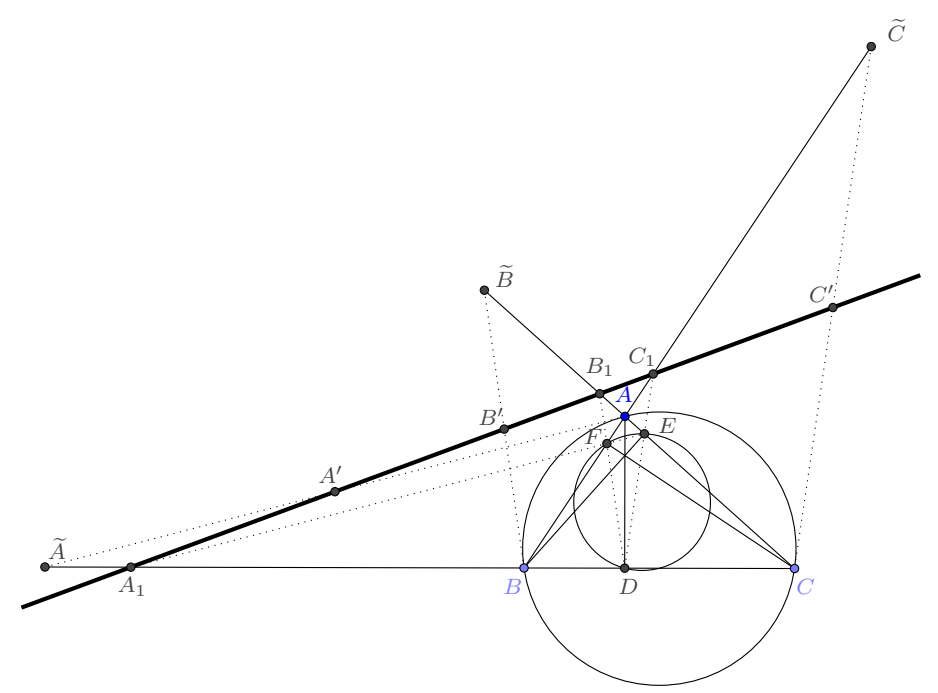

Figura 24: La línea gruesa es el eje radical del circuncírculo y la circunferencia de Feuerbach.

cuncírculo y la circunferencia de los nueve puntos es la recta de Euler. Sobre esta recta se encuentran el ortocentro, el circuncentro, el centro de la circunferencia de los nueve puntos, el gravicentro, y algunos otros puntos notables del triángulo [3]. En consecuencia, los centros de las circunferencias de la familia del circuncírculo pertenecen a la recta de Euler.

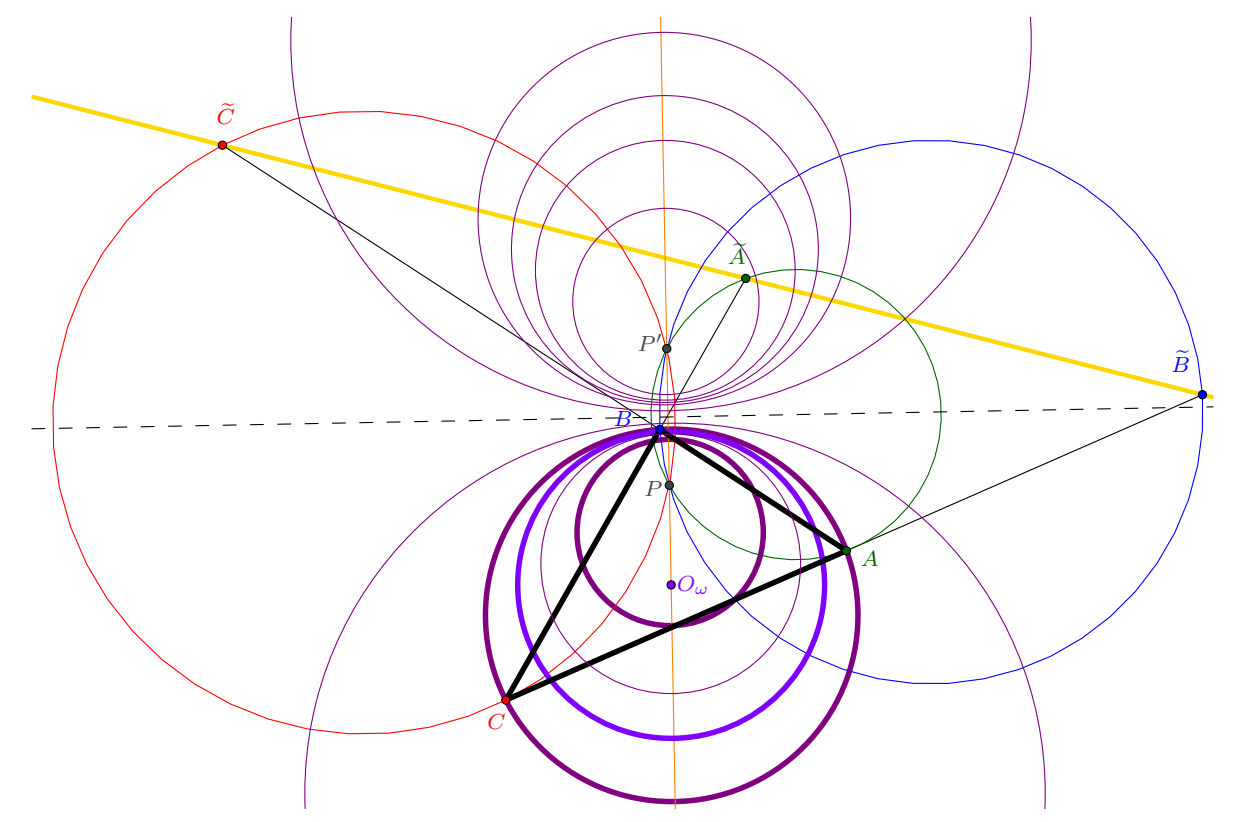

Figura 25: En amarillo, la recta generada por el circuncírculo. En morado, el circuncírculo y la circunferencia de los nueve puntos de $\triangle A B C$ y otros miembros de la misma familia, entre ellos los puntos $P$ y $P^{\prime}$ que son de radio cero. La línea punteada es el eje radical común y en color naranja tenemos la recta de Euler. Las circunferencias $\mathscr{C}_{A}$ (verde), $\mathscr{C}_{B}$ (azul) y $\mathscr{C}_{C}$ (rojo) son ortogonales a cada circunferencia en la familia.

Sea $\omega$ una circunferencia en la familia anterior (color violeta). Sabemos que su centro 
$O_{\omega}$ pertenece a la recta de Euler, y que $\omega$ es ortogonal a $\mathscr{C}_{A}, \mathscr{C}_{B}$ y $\mathscr{C}_{C}$. Sean $P$ y $P^{\prime}$ los puntos comunes de $\mathscr{C}_{A}, \mathscr{C}_{B}$ y $\mathscr{C}_{C}$. Como $\omega$ es ortogonal a ellas, su radio $R_{\omega}$ es igual a la raíz cuadrada de la potencia de $O_{\omega}$ con respecto a esas circunferencias: $R_{\omega}^{2}=O_{\omega} P \cdot O_{\omega} P^{\prime}$. En particular, la potencia de $O_{\omega}$ respecto a dichas circunferencias es positiva, por lo que $O_{\omega}$ está en la recta de Euler pero fuera del segmento $P P^{\prime}$.

Por ejemplo, el ortocentro $H$ tiene potencia negativa respecto a $\mathscr{C}_{A}, \mathscr{C}_{B}, \mathscr{C}_{C}$. Por ello, no existe circunferencia centrada en $H$ que pertenezca a la familia del circuncírculo. En cambio, como el gravicentro $G$ está entre el circuncentro y el centro de la circunferencia de los nueve puntos, se encuentra fuera del segmento $P^{\prime} P$ y sí existe una circunferencia centrada en el gravicentro que pertenece a la familia.

\section{Reformulaciones del Teorema 2.1}

El siguiente resultado (Teorema 5.2) es una reformulación del Teorema 2.1, en el sentido de que ambos resultados son equivalentes. Dicha reformulación se obtiene por la dualidad entre las nociones de polos y polares.

Dadas una circunferencia $\omega$ y una recta $\mathscr{L}$ en el plano, se define el polo de $\mathscr{L}$ como el punto $P$ tal que $\mathscr{L}_{P}=\mathscr{L}$, es decir, tal que $\mathscr{L}$ es la polar de $P$ respecto a $\omega$. En particular, $O$ es el polo de la línea al infinito y las rectas por $O$ tienen un punto al infinito por polo.

La Proposición 1.2, en términos de la noción de polo, se reformula como sigue:

Proposición 5.1 Sean $\omega$ una circunferencia, $\mathscr{L}, \mathscr{M}, \mathscr{N}$ rectas en el plano y $P_{\mathscr{L}}, P_{\mathscr{M}}, P_{\mathscr{N}}$ sus respectivos polos. Se tiene lo siguiente:

- Si $\mathscr{L}$ pasa por $P_{\mathscr{M}}$, entonces $\mathscr{M}$ pasa por $P_{\mathscr{L}}$.

- Si $\mathscr{N}$ es la recta que pasa por $P_{\mathscr{L}}$ y $P_{\mathscr{M}}$, entonces $P_{\mathscr{N}}$ es la intersección de $\mathscr{L}$ y $\mathscr{M}$.

- Las rectas $\mathscr{L}, \mathscr{M}, \mathscr{N}$ son concurrentes si y sólo si $P_{\mathscr{L}}, P_{\mathscr{M}}, P_{\mathscr{N}}$ son colineales.

En términos de la noción de polo, el Teorema 2.1 equivale al siguiente:

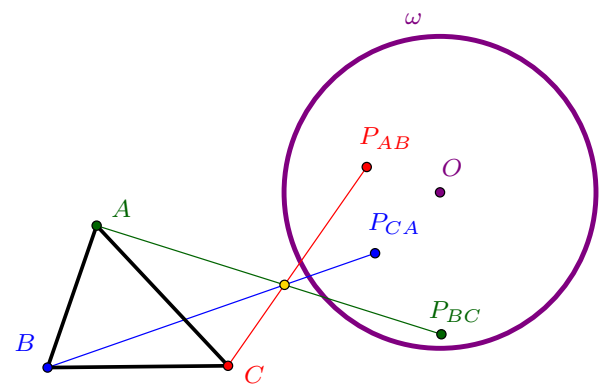

Figura 26: El dual del Teorema 2.1 utilizando polos de rectas. 
Teorema 5.2 Sean $\triangle A B C$ un triángulo y $\omega$ una circunferencia en el plano. Sean $P_{B C}$, $P_{C A}$ y $P_{A B}$ los polos de las rectas $B C, C A$ y $A B$ respecto a $\omega$. Se tiene que las rectas $A P_{B C}$, $B P_{C A}$ y $C P_{A B}$ son concurrentes.

Prueba. Por la Proposición 5.1, demostrar la concurrencia de las rectas $A P_{B C}$ (verde), $B P_{C A}$ (azul), $C P_{A B}$ (roja) es equivalente a probar que sus polos son colineales. Sean $\widetilde{A}$, $\widetilde{B}, \widetilde{C}$ como en el enunciado del Teorema 2.1. Por un lado, por la Proposición 5.1, el polo de $A P_{B C}$ es la intersección de las rectas polares $A$ y de $P_{B C}$. La recta polar de $P_{B C}$ es precisamente $B C$, por lo cual, el polo de la recta $A P_{B C}$ es $\widetilde{A}$. Similarmente, el polo de $B P_{C A}$ es $\widetilde{B}$ y el polo de $C P_{A B}$ es $\widetilde{C}$. Por el Teorema 2.1, $\widetilde{A}, \widetilde{B}, \widetilde{C}$ son colineales. Esto significa que sus polares $A P_{B C}, B P_{C A}, C P_{A B}$ son concurrentes, como queríamos probar.

Como comentábamos arriba, los Teoremas 2.1 y 5.2 son equivalentes. Se invita al curioso lector a tratar de generalizar el Teorema 5.2 a dimensiones superiores. Por ejemplo, dada una esfera $\omega$ en el espacio de dimensión 3, el polo de un plano es un punto, la polar de una recta es otra recta y el polo de un punto es un plano. En estos términos, puede presentarse una formulación $n$-dimensional del Teorema 5.2 y tratar de obtenerse una demostración.

Procedemos ahora a presentar una última reformulación del Teorema 2.1, utilizando secciones cónicas. Recordemos que el Teorema 2.1 es un resultado de geometría proyectiva, lo cual garantiza que dicho resultado sigue siendo válido si las rectas polares se toman con respecto a cualquier cónica. La noción de recta polar respecto a una cónica en general no la discutiremos aquí, sólo hay que entender que la Proposición 1.3 es válida si cambiamos circunferencia por cónica. De esta manera, la recta polar $\mathcal{L}_{X}$ del punto $X$ respecto a una cónica $\Gamma$ puede construirse como sigue: se trazan dos rectas por $X$ que corten a $\Gamma$ en cuatro puntos $P, Q, R, S$, y luego se toman los puntos $Y$ y $Z$ como la intersección de las diagonales y/o lados opuestos del cuadrilátero $P Q R S$. Resulta que recta determinada por los puntos $Y$ y $Z$ es siempre la mimsa para el punto $X$, de manera que $Y Z$ se define como la recta polar de $X$ respecto de $\Gamma$ (ver [2]).

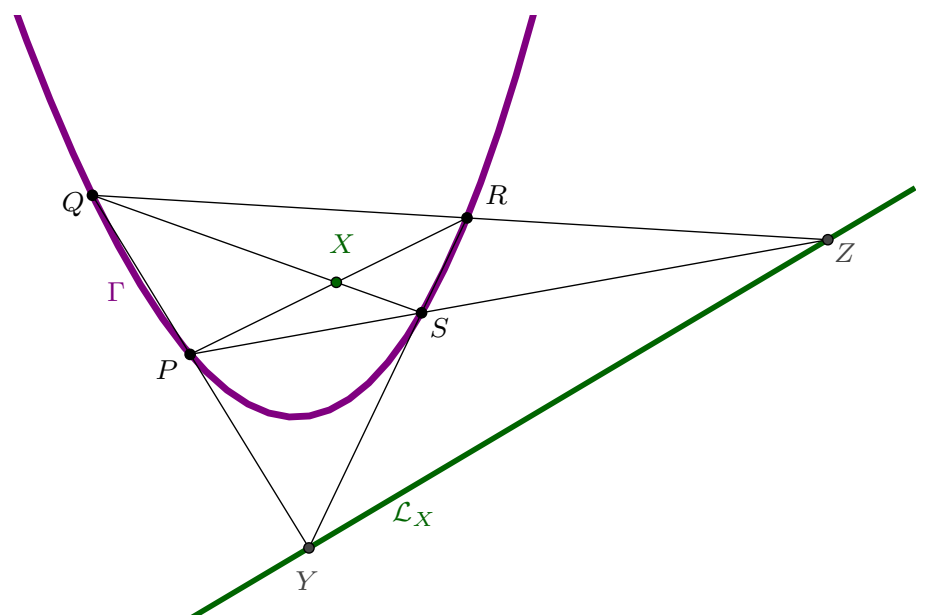

Figura 27: Construyendo la recta polar de $X$ respecto a la parábola $\Gamma$. 
Sea $\omega$ una circunferencia en el plano proyectivo $\mathbb{R P}^{2}$, es decir, el plano con la línea al infinito. Consideremos otro plano proyectivo $\mathbb{R P}^{2}$ y sea $\Gamma$ una cónica en dicho plano. Vamos exhibir una transformación proyectiva $\pi: \mathbb{R} P^{2} \rightarrow \mathbb{R P}^{2}$ que transforma la cónica $\Gamma$ en la circunferencia $\omega$. Para ello, apelaremos a la definición de sección cónica. Fijemos un cono circular recto en el espacio proyectivo $\mathbb{R P}^{3}$, o sea, en el espacio con el plano al infinito, como en la Figura 28. Tomemos el plano proyectivo $\mathbb{R P}^{2}$ que contiene a $\omega$ (azul) e intersequémoslo con el cono, de manera que su intersección sea precisamente $\omega$. De la misma manera, tomemos el plano proyectivo $\mathbb{R P}^{2}$ que contiene a $\Gamma$ (verde) e intersequémoslo con el cono, de manera que su intersección sea precisamente $\Gamma$.

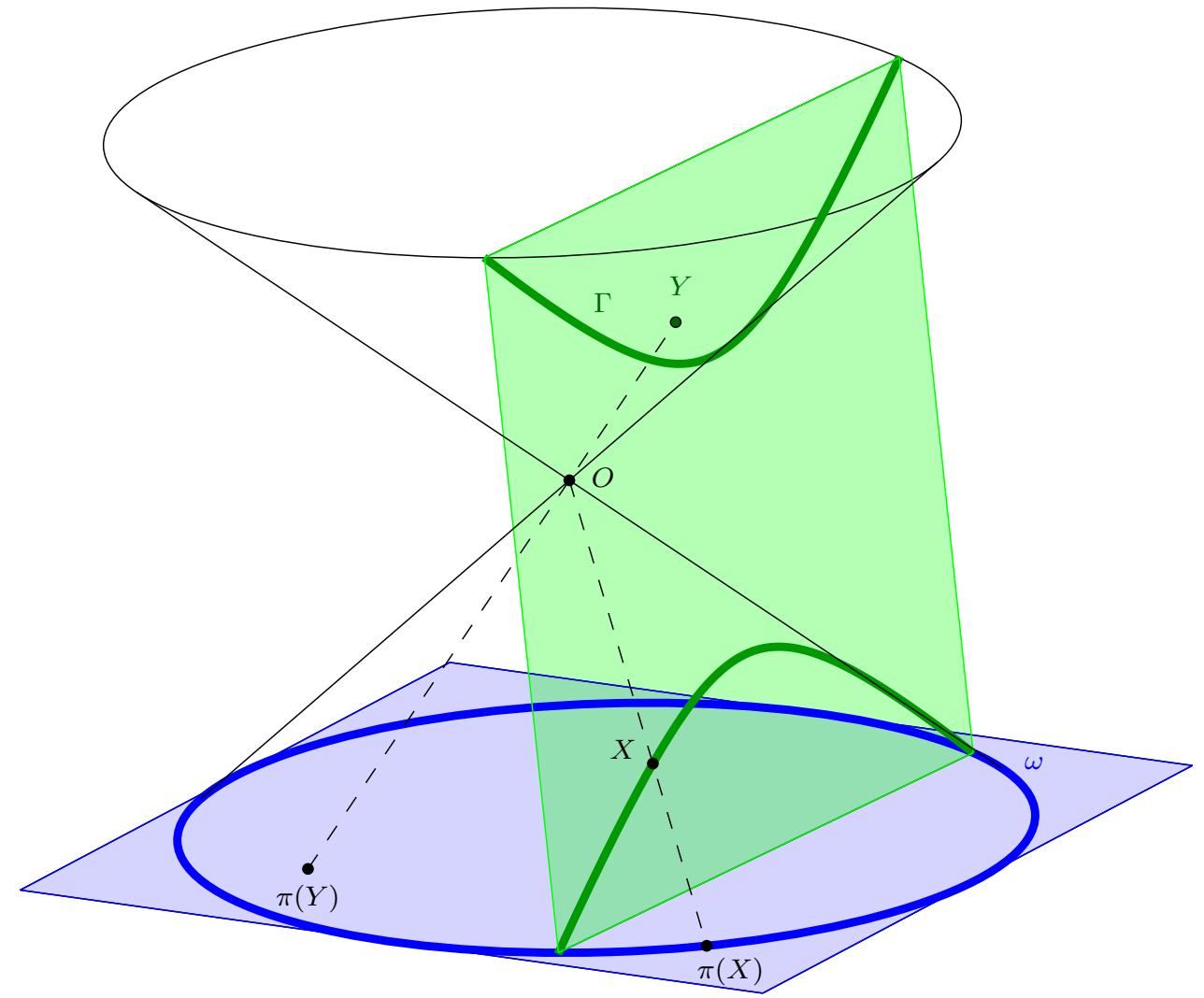

Figura 28: $\Gamma$ es una hipérbola en el plano verde, $\omega$ una circunferencia en el plano azul y $\pi$ es una transformación proyectiva entre ambos planos que es una biyección de $\Gamma$ en $\omega$.

Ahora, para cada punto $X$ del plano proyectivo de $\Gamma$ (verde), tomemos la recta $X O$, donde $O$ es el vértice del cono, y prolonguémosla hasta intersecar al plano de $\omega$ (azul). A su intersección la denotamos por $\pi(X)$, por lo que se tiene definida una correspondencia $\pi: \mathbb{R} P^{2} \rightarrow \mathbb{R P}^{2}$. No es difícil convencerse que esta transformación envía puntos de $\Gamma$ a puntos de $\omega$, ya la imagen de puntos en el cono sigue perteneciendo al cono. Además, cada punto de $\omega$ es la imagen de un único punto $X$ de $\Gamma$, pudiendo ser $X$ un punto al infinito (recordemos que los planos son proyectivos, y contienen la línea al infinito). Más aún, $\pi: \mathbb{R} P^{2} \rightarrow \mathbb{R} P^{2}$ transforma puntos colineales en puntos colineales, y similarmente, se puede demostrar que transforma rectas tangentes a $\Gamma$ en rectas tangentes a $\omega$. 
Teorema 5.3 Sean $\triangle X Y Z$ un triángulo y $\Gamma$ una cónica en el plano proyectivo $\mathbb{R P}^{2}$. Sean $\mathcal{L}_{X}, \mathcal{L}_{Y}$ y $\mathcal{L}_{Z}$ las rectas polares de $X, Y$ y $Z$, respecto de $\Gamma$. Sean $\bar{X}$ la intersección de $Y Z$ con $\mathcal{L}_{X}, \bar{Y}$ la intersección de $Z X$ con $\mathcal{L}_{Y}, \bar{Z}$ la intersección de $X Y$ con $\mathcal{L}_{Z}$. Se tiene que los puntos $\bar{X}, \bar{Y}$ y $\bar{Z}$ son colineales.

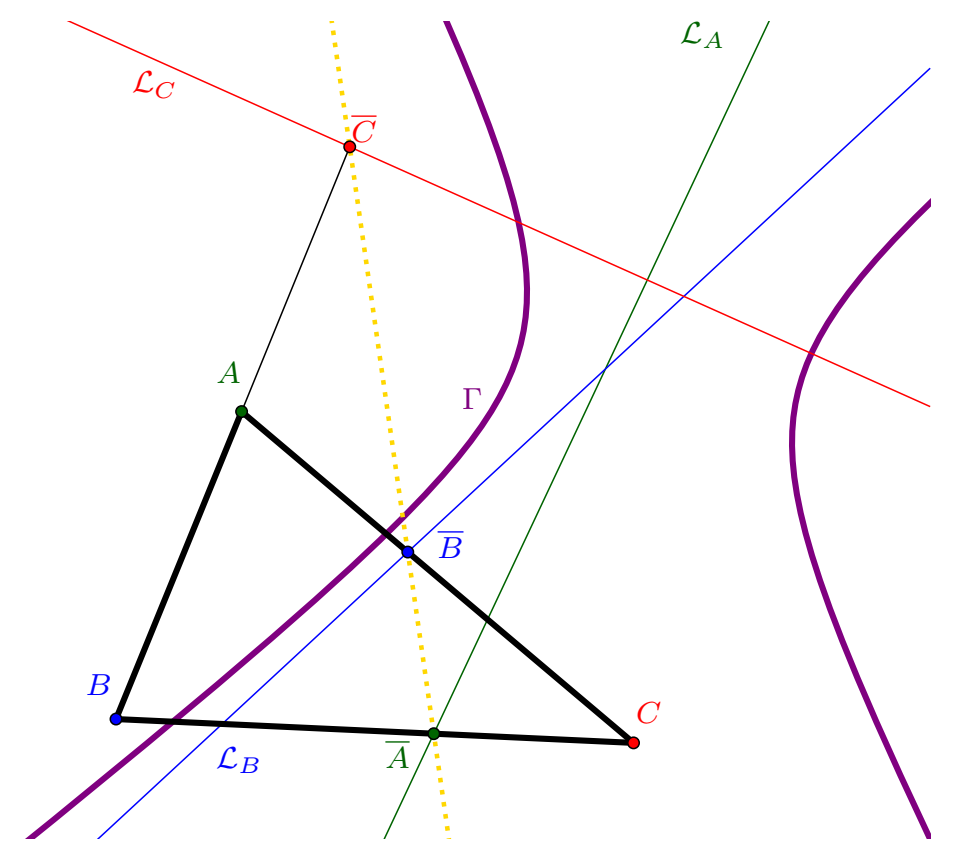

Figura 29: Repitiendo la construcción del Teorema 2.1 utilizando cualquier cónica.

Prueba. Sea $\omega$ una circunferencia en el plano proyectivo $\mathbb{R P}^{2}$ y sea $\pi: \mathbb{R} P^{2} \rightarrow \mathbb{R P}^{2}$ la trasformación proyectiva descrita anteriormente. Denotemos por $A=\pi(X), B=\pi(Y), C=$ $\pi(Z)$ a la imagen de los vértices del triángulo. Para cada $P$ en el plano de $\omega$, denotemos por $\mathscr{L}_{P}$ a la recta polar de $P$ respecto de $\omega$. Puesto que $\pi$ preserva concurrencia y colinealidad, se tiene que las rectas polares respecto a $\Gamma$ son enviadas en las polares respecto a $\omega$ :

$$
\pi\left(\mathcal{L}_{X}\right)=\mathscr{L}_{A}, \quad \pi\left(\mathcal{L}_{Y}\right)=\mathscr{L}_{B}, \quad \pi\left(\mathcal{L}_{Z}\right)=\mathscr{L}_{C}
$$

Sean ahora $\widetilde{A}, \widetilde{B}, \widetilde{C}$ los puntos definidos como en el enunciado del Teorema 2.1, es decir,

$$
\widetilde{A}=\mathscr{L}_{A} \cap B C, \quad \widetilde{B}=\mathscr{L}_{B} \cap C A, \quad \widetilde{C}=\mathscr{L}_{C} \cap A B .
$$

De nuevo, como $\pi$ preserva colinealidad, se tiene que $\pi(X Y)=A B, \pi(Y Z)=B C$ y $\pi(Z X)=$ $C A$. Por ello, se tiene también que

$$
\begin{aligned}
& \pi(\bar{X})=\pi\left(\mathcal{L}_{X} \cap Y Z\right)=\mathscr{L}_{A} \cap B C=\widetilde{A}, \\
& \pi(\bar{Y})=\pi\left(\mathcal{L}_{Y} \cap Z X\right)=\mathscr{L}_{B} \cap C A=\widetilde{B}, \\
& \pi(\bar{Z})=\pi\left(\mathcal{L}_{Z} \cap X Y\right)=\mathscr{L}_{C} \cap A B=\widetilde{C} .
\end{aligned}
$$

Por el Teorema 2.1. los puntos $\widetilde{A}, \widetilde{B}, \widetilde{C}$ son colineales, es decir, los puntos $\pi(\bar{X}), \pi(\bar{Y})$, $\pi(\bar{Z})$ son colineales. Puesto que $\pi$ es proyectiva, se tiene que $\bar{X}, \bar{Y}, \bar{Z}$ son colineales, como queríamos. 


\section{Referencias}

[1] Howard Eves, Estudio de las Geometrías, Tomo I, 471, UTEHA, Méxcio (1969).

[2] Jeremy Gray, Worlds Out of Nothing. A Course in the History of Geometry in the 19th Century, 384, Springer, London (2010).

[3] Levi S. Shively, Introducción a la Geometría Moderna, 173, Editorial CECSA, México (1972). 